\title{
The Development of a State-of-the-Art Assay System for Uranium-235 in Solutions
}

\author{
J. L. Parker \\ T. E. Sampson \\ L. R. Cowder \\ E. A. Kern \\ D. L. Garcia \\ N. Ensslin
}

\author{
DISCLAIMER
}

\begin{abstract}
This report was prepared as an account of work sponsored by an agency of the United States Government. Neither the United States Government nor any agency thereof, nor any of their employees, makes any warranty, express or implied, or assumes any legal liability or responsibility for the accuracy, completeness, or usefulness of any information, apparatus, product, or process disclosed, or represents that its use would not infringe privately owned rights. Reference herein to any specific commercial product, process, or service by trade name, trademark, manufacturer, or otherwise does not necessarily constitute or imply its endorsement, recommendation, or favoring by the United States Government or any agency thereof. The views and opinions of authors expressed herein do not necessarily state or reflect those of the United States Government or any agency thereof.
\end{abstract}

\section{MASTER}

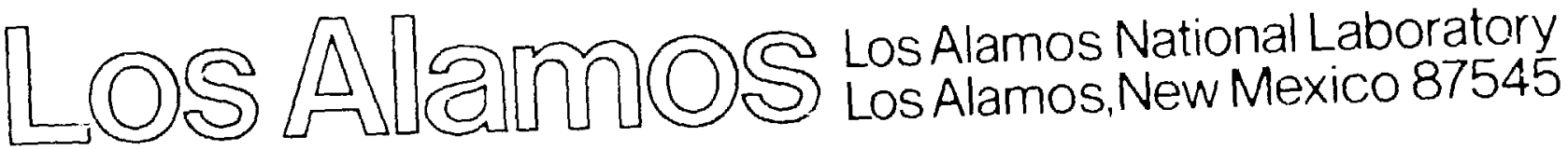


ABSTRACT

I. INTRODUCTION . . . . . . . . . . . . . . . 1

II. PRevious State of The ART... . . . . . . . . . 2

III. SYSTEM DESIGN AND PERFORMANCE PARAMETERS . . . . . . 2

IV. INITIAL PERFORMANCE ESTIMATES . . . . . . . . . . . . 3

V. TERFORMANCE DEVELOPMENT................. . . . 4

A Counting Configuration . . . . . . . . . . . . . 4

B. Sample Vial Characteristics . . . . . . . . . 7

C. Electronic Improvements . . . . . . . . . . . . 9

D. Count-Time Optimization . . . . . . . . . . . . 11

E. Background Reduction............. . 12

F. Transmission Correction . . . . . . . . . . . 15

G. Solution Depth Measurement . . . . . . . . 15

VI. PERFORMANCE TESTING . . . . . . . . . . . . 15

A. High-Level Detectors... . . . . . . . . . . . 15

B. Calibration Curve Nonlinearity. . . . . . . 19

C. Low-Level Detectors . . . . . . . . . . . . 22

D. Measurement Precision ............. . . 23

E. Count-Rate Bias Effects . . . . . . . . . 23

VII. SUMMARY . . . . . . . . . . . . . . . 25

APPENDIX A: FEATURES OF THE SYSTEM . . . . . . . . . 28

APPENDIX B: ALGORITHMS FOR COMPUTATION OF TOTAL

CORRECTED RATE............... . . 31

APPENDIX C: SYSTEM PHOTOS ............... 35

REFERENCES . . . . . . . . . . . . . . . . . . 39 
THE DEVELOPMENT OF A STATE-OF-THE-ART ASSAY SYSTEM

FOR URANIUM-235 IN SOLUTIONS

by

J. L. Parker, T. E. Sampson, L. R. Cowder

E. A. Kern, D. L. Garcia, and N. Enssiin

\begin{abstract}
We describe the development of a high-accuracy, highprecision, and high-throughput system for the assay of $235 \mathrm{U}$ in solution samples. The tradeoffs involved in the various development steps are discussed and the ultimate system performance is documented. Assay accuracy and precision better than $0.2 \%$ should be attained in routine use. This is a significant improvement in the state of the art.
\end{abstract}

\title{
I. INTRODUCTION
}

In 1982, the Los Alamos National Laboratory Safeguards Assay Group (Q-1) was asked to develop a new assay system for ${ }^{235} \mathrm{U}$ in solution form; successful completion of the project required a significant advancement in the state cif the art.

We chose transmission-corrected passive gamma-ray assay of the 185.7-keV gamma ray from ${ }^{235} \mathrm{U}$ as the measurement technique, with a ${ }^{169} \mathrm{Yb}$ source for transmission measurements and an ${ }^{241}$ Am source for rate-loss corrections.

This report describes the system's performance and development. The report assumes a familiarity with the principles of transmission-corrected passive gamma-ray assay applied to ${ }^{235} \mathrm{U}$ solutions. Appendixes to this report present specifications and hardware features. 


\section{PREVIOUS STATE OF THE ART}

Solution assay systems for uranium and plutonium have been developed and used at Los Alamos for over a decade. The procedures and techniques are well understood and thoroughly tested. When this project was planned a uranium solution assay system (USAS) ${ }^{1}$ had been in routine use for seven years at the Los Alamos uranium recovery facility.

The transmission-corrected passive assay portion of the USAS operated over the narrow concentration range of $2-50 \mathrm{~g} / \ell$, with measurement precision of 0.7-0.3\% relative standard deviation (RSD) for 400-s count times. Measurement bias ranged from 0.2-1.0\% depending upon the elapsed time since recalibration.

Lower concentration solutions $(0.001-0.5 \mathrm{~g} / \mathrm{l})$ were measured with an accuracy of around 10\%. The poorer accuracy resulted from sensitivity to matrix effects because the measurement was not transmission corrected.

The USAS showed the value of the transmission-corrected passive assay technique for routine operation in a production facility when operated by skilled and conscientious personnel.

\section{SYSTEM DESIGN AND PERFORMANCE PARAMETERS}

The design and performance parameters provided by the user that influenced system design are listed below:

$\begin{array}{ll}235 \mathrm{U} \text { Concentration Range: } & 0.1 \mathrm{mg} / \mathrm{\ell} \text { to } 500 \mathrm{~g} / \mathrm{l} \\ \text { Sample size } & 30 \mathrm{m \ell}-1 \text { to } 500 \mathrm{~g} / \mathrm{\ell} \\ & 200 \mathrm{m \ell}-0.1 \mathrm{mg} / \mathrm{\ell} \text { to } 10 \mathrm{~g} / \mathrm{l} \\ \text { Counting time } & 1000 \mathrm{~s} \text { per sample, all samples } \\ \text { Accuracy and precision }: & 0.1 \% \text { relative, for high concentrations } \\ \text { Sample vial } & : \text { commercial source } \\ \text { Sample filling } & : \text { automatic fillers, thus a variable sample depth } \\ \text { Sample transport } & : \text { pneumatic "rabbit" system }\end{array}$

Some comments on these requirements will put the design problem in better perspective. The $235 \mathrm{U}$ concentration range is nearly seven orders of magnitude. This wide dynamic range interacted with the user's desire to provide minimum sample volumes (to reduce waste) and resulted in a system with two 
measurement heads (detectors). One detector measures "high-level" or more concentrated solutions in a 30-me sample (determined by the sample size needed by the plant's analytical chemistry facility); the "low-level" or low-concentration measurements are made on 200-me samples, which allow bette, counting precision than the 30-me samples. Both of these sample sizes are smaller than what we desired but were required by the user.

The counting time of 1000 s naturally influences the attainable statistical precision. Increasing the counting time would improve the statistical precision for all concentrations. However, we did attain (barely) the $0.1 \%$ RSD precision for high-concentration solutions (>100 g/l). The goal of $0.1 \%$ accuracy (defined here as the difference between the measured value and the true value) is strongly influenced by the consistency of the calibration standaras available. Inconsistent calibration standards were one of several major problems encountered duririg the program.

The jroperties of the sample vials also influence the accuracy and precision. High precision requires the highest possible sample counting rates, which means small sample-to-detector distances (a few cm for high-level solutions, less for low-level solutions). The small distances increase the sensitivity of the assay to variations in sample dimensions and sample position arising from manufacturing tolerances in the sampie vial dimensions.

For material strength and resistance to chemical attack, the sample vials were manufactured from polypropylene. However, polypropylene has less rigidity and dimensional stability than some other plastics; $1 / r^{2}$ effects from sample vial dimensional variations increased problems with assay accuracy.

variable sample depths posed another difficult problem. The user chose an automatic sample filling system, which reduces labor costs over the alternative of accurate pipetting. but causes variable sample depths. The transmission-corrected passive assay technique can handle this problem if the sample depth is either constant or known (see section V.F.). A separate device was needed to accurately measure the sample depth. The development of this depth gauge was contracted to a well-known firm in the ultrasonic measurement field.

IV. INITIAL PERFORMANCE ESTIMATES

The requirements and goals for system performance desired by the user have been outiined in Sec. III, and the difficulties in attaining all of these goals 
have been mentioned. At the project onset we estimated what the performance might be in a system designed to meet those goals and with the constraints set out in $\sec$. III. These initial estimates are given in Table I.

These estimates did not meet the goals. We expended several staff years of $R \& D$ to push the estimated performance toward the goals. Some of the steps taken to improve system accuracy and precision are discussed in the following sections.

\section{PERFORMANCE DEVELOPMENT}

\section{A. Counting Configuration}

The first decision was to choose the counting configuration. The two choices were an up-looking geometry or a side-looking geometry (Fig. 1). These two configurations measure fundamentaliy different quantities.

The up-looking geometry views the bottom of a cylindrical solution sample, viewing the entire solution volume. It measures the ${ }^{235} \mathrm{U}$ mass in the total solution volume. Coupled with a mass measurement of the solution, it yields a result in $\mathrm{g}{ }^{235} \mathrm{U} / \mathrm{g}$ sample. With g ${ }^{235} \mathrm{U} / \mathrm{g}$ sample determined, the batch mass is required to determine the batch ${ }^{235} \mathrm{U}$ mass. If dip tube manometry is used to measure the batch solution depth (batch volume being a function of batch depth), the batch density is also determined. The batch mass is then the product of the batch volume and the batch density.

The side-looking geometry views a collimator-defined portion of solution contained in a parallel-sided vial. Because all of the solution is not viewed,

\section{TABLE I}

ESTIMATED ACCURACY FOR ASSAY OF $235 \mathrm{U}$ IN HIGH-CONCENTRATION SOLUTIONS

Single wide-range 1-500 g/2 calibration

$0.5-1.0 \% *$

Narrow-range 50-300 g/e calibration

$0.2-0.3 \%$ *

*Instrument improvements, a depth measuring device, and more precisely manufactured sample vials may reduce these estimates. 


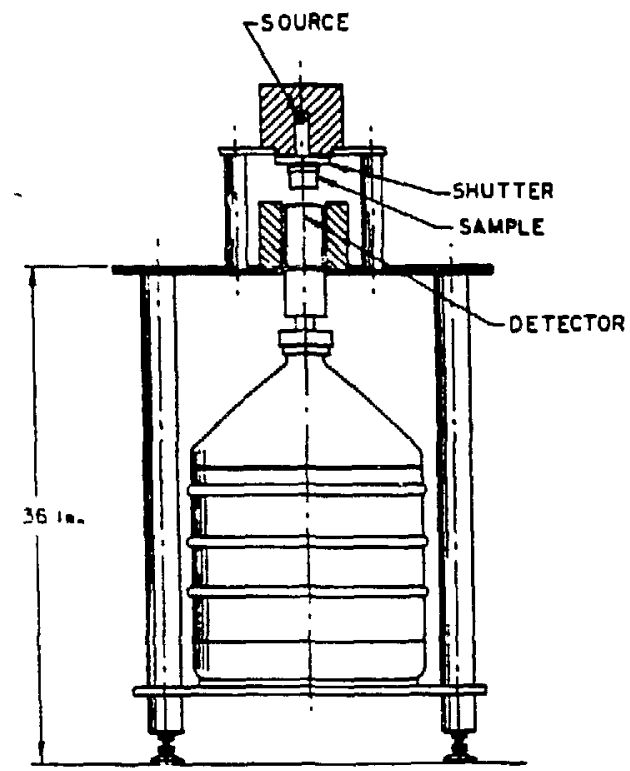

(a)

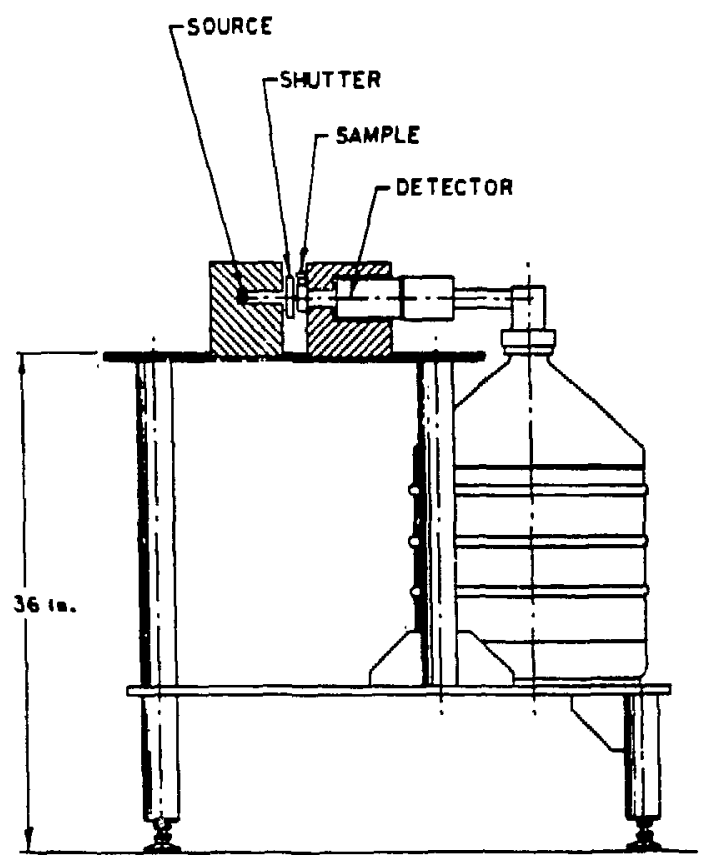

(b)

Fig. 1. High-level solution assay geometry: (a) uplooking and (b) sidelooking.

this technique has to be calibrated in terms of solution concentration, $g{ }^{235} \mathrm{U} / \mathrm{R}$. The dip tube marometry measurement of batch volume can be used directly to get the batch ${ }^{235} \mathrm{U}$ mass.

Advantages of up-looking geometry $\left(g^{235} \mathrm{U} / \mathrm{g}\right.$ sample)

1. The entire sample is viewed, providing the most efficient use of the limited sample volume and the best statistical precision for a given total sample size, as much as a factor of 2 better than the alternative.

2. A $1 \%$ deviation from the stated sample depth results in $0.15-0.25 \%$ deviations in the ${ }^{235} \mathrm{U}$ assay for sample-to-detector distances of $5 \mathrm{~cm}$ to $2 \mathrm{~cm}^{2}$ For a $3-\mathrm{cm}$ sample-to-detector distance, the sample depth variation must be less than 0.003 in. to keep assay errors from this effect below $0.1 \%$. This assumes a $20 \mathrm{~cm}^{2} \times 1.5-\mathrm{cm}$ deep sample (30 $\mathrm{m} \ell$ ).

3. The sample and batch temperatures may be different. Only total mass is being measured. 
4. The cylindrical bottle is easter to fabricate to required tclerances than the flat bottle of the alternative approach.

5. Geometry is more compact, taking less plant space.

Disadvantages of up-looking geometry (g $235 \mathrm{U} / \mathrm{g}$ sample)

1. The sample depth and diameter must be fixed or known. The sample-todetector distance must be constant. For a 3 -cm distance, a $1 \%$ change in the distance results in about $0.8 \%$ change in the assay. This sensitivity is the geometry's biggest disadvantage and means that sampleto-detector distances must be held to about \pm 0.001 in. to keep the assicy error below $0.1 \%$.

2. Sample depth must be accurately measured or fixed by careful pipetting.

3. Sample transport through the pneumatic rabbit may cause some solution to "hang up" in the threads because of a poor seal. This can perturb the sample geometry and cause an assay error.

Advantages of side-looking geometry $\left(g^{235} \mathrm{U} / \ell\right)$

1. Ease of sampling is the biggest advantage. The sample volume needs only exceed that required to fill the volume defined by the collimator.

2. Small variations in the sample-to-collimator distance have little effect on the assay.

Disadvantages of side-looking geometry $\left(g^{235} \mathrm{U} / \mathrm{\ell}\right)$

1. Less efficient use of limited sample volume gives poorer precision than alternative method.

2. Sample thickness must be constant or known. Assay error is approximately 1:l with sample thickness errur. Thus thickness tolerances must be on the order of \pm 0.001 in. to keep the assay error from this effect below $0.1 \%$.

3. Corrections must be made for any temperature differences between the sample and the batch. Differences of a few tens of degrees celsius can lead to errors of several tenths of a percent. Expansion coefficients as well as temperatures must be known to correct this effect. 
We carefully examined the above advantages and disadvantages and chose the up-looking ( ${ }^{235} \mathrm{U} / \mathrm{g}$ sample) geometry, primarily because it offered the only possibility of attaining $0.1 \%$ RSD precision in 1000-s count times for $>100$ $\mathrm{g} / \mathrm{e}$ solutions. This requirement was assigned great importance by the user.

\section{B. Sample vial Characteristics}

After choosing the counting configuration we specified sample vial dimensions. These systems are most efficient when the greatest sample volume is closest to the detector, but the sample diameter should not greatly exceed the detector crystal diameter. Thus we chose the sample diameter to be nearly Equal to the detector diameter and accepted the sample depth that came with it.

Sample solution dimensions for the high-level $(1-500 \mathrm{~g} / \ell)$ system are $1.99 \mathrm{in.} \operatorname{diam}\left(20 \mathrm{~cm}^{2}\right) \times 0.59 \mathrm{in}$. deep $(1.5 \mathrm{~cm})$, giving a volume of $30 \mathrm{ml}$. This matrhes the nominal 50-mm-diam detector used for high-level solutions.

The low-level solution diameter is $2.63 \mathrm{in.}(6.68 \mathrm{~cm})$, somewhat exceeding the $5.8-\mathrm{cm}$ detector diameter. Solution depth is $5.71 \mathrm{~cm}$, giving a volume of $200 \mathrm{ml}$.

Bottles are made of polypropylene with screw-top lids, with bottom thickness for both high-and low-level bottles of 0.060 in.

We carried out extensive tests on pilot runs of the commercially manufactured bottles, carefully measuring the deviation from flatness in the bottle bottoms. Variations in this flatness can cause assay errors because of the changing solution-to-detector distance. Figure 2 shows the measurement positions on the bottle bottom (a) and the results of a typical scan (b) for a high-level bottle. Typical bowing in the center of the bottle bottom is 0.004-0.005 in., but is not expected to be a problem unless it changes from bottle to bottle. Figure 3 shows the distribution of the average bowing for a sample of 25 bottles. The average bowing is defined by the six points for each scan (three on each side of the center) in scans 1 and 11 . We do not expect the distribution from -0.002 to $-0.005 \mathrm{in}$. to be a problem. However, the two outliers around +0.007 in. could be a problem. If we assume that the bowing displaces about one-third of the solution volume (solution near the periphery is little affected by the bowing), then the approximate assay error from the outlier bottles is

bowing $(0.001$ in. $) \times 1 / 3 \times 0.08 \% \frac{\text { error }}{0.001}$ in. 


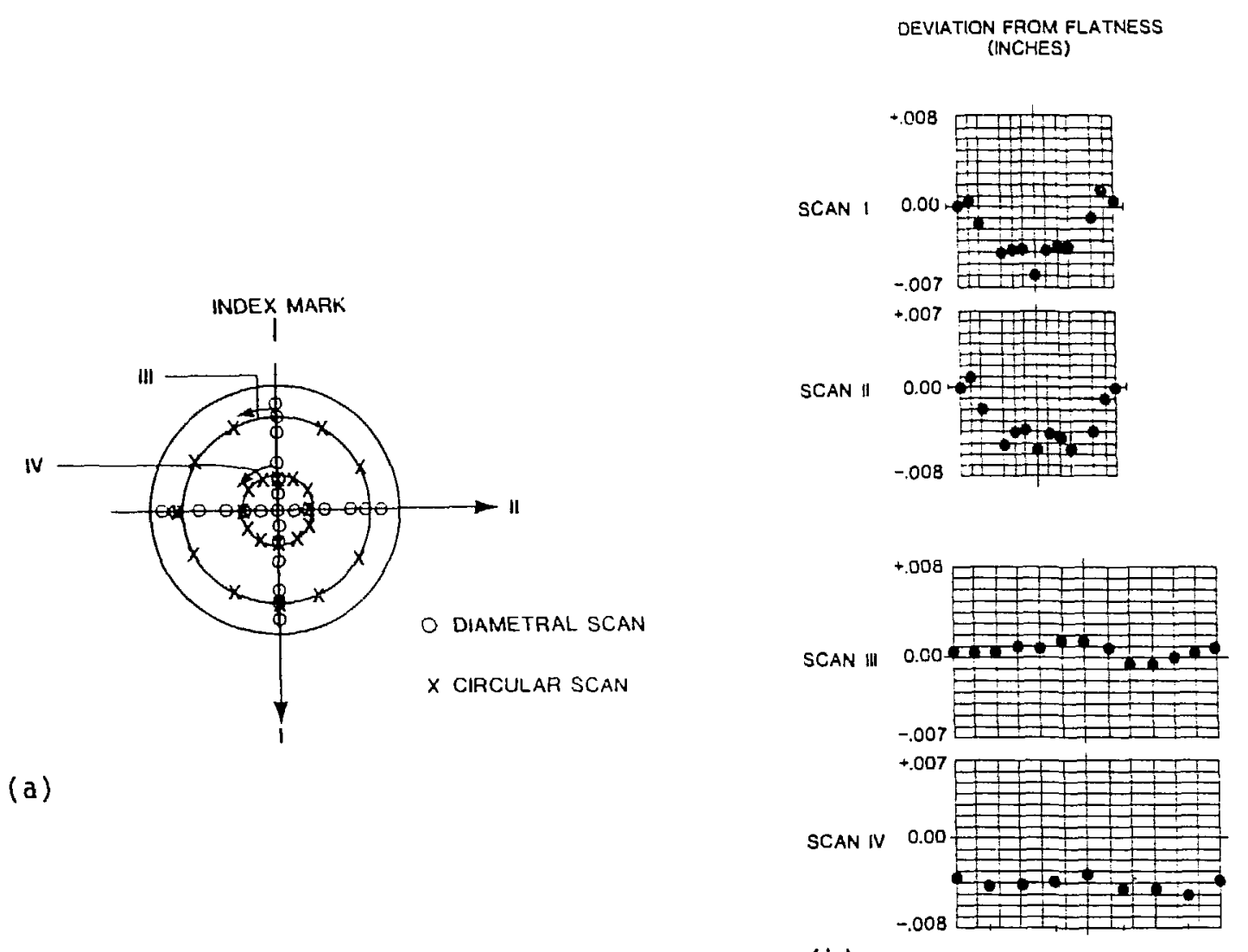

(b)

Fig. 2. Bowing measurements (deviation from flatness in inches) on a typical high-level sample vial: (a) scan pattern and (b) scan results.

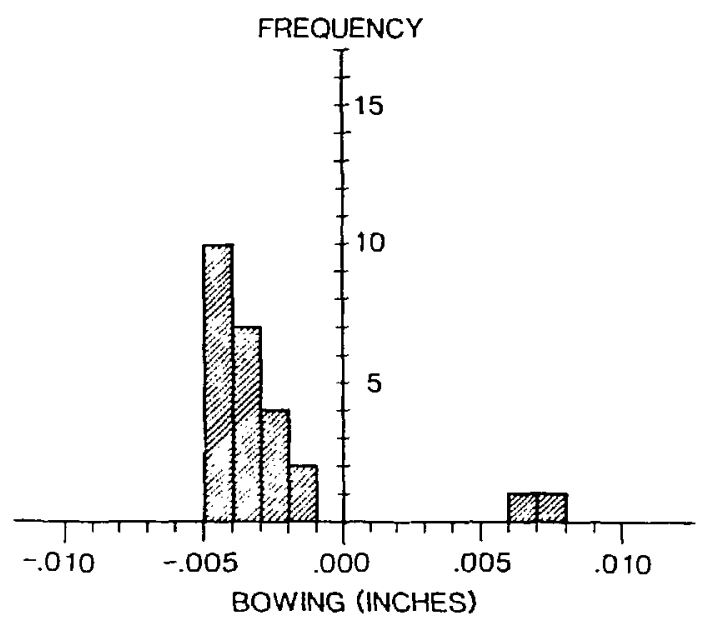

Fig. 3. Distribution of bowing for a sample of 25 bottles for high-level measurements. 
where bowing is the difference between the bowing in the standards and in the sample. Here, the difference between the main body of bottles and the outliers is $\sim 0.011 \mathrm{in.}$ Reference 2 provides a $0.08 \%$ error $/ 0.001 \mathrm{in}$. for a 3-cm sampleto-detector distance, giving $11 \times 0.33 \times 0.08=0.29 \%$ assay error. This error could be significant when compared with the requested accuracy of $0.1 \%$ and points out the need for strict quality assurance testing on the manufactured botties.

We also measured the transmission of the empty bottles at $186 \mathrm{keV}$ to determine if variations would significantly affect the sample transmission measurement. These results are given in Table II.

The standard deviation coiresponds to a relative assay error of about $0.06 \%$. A $1 \%$ error in the transmission corresponds to about a $0.4 \%$ error in the sample assay. Variations in bottle thickness affect the transmission measurement, causing some assays to have errors as large as $0.1 \%$. It is clear that the sample vials limit our ability to attain $0.1 \%$ accurate assays.

\section{Electronic Improvements}

Electronic improvements and performance optimization were needed to preserve measurement accuracy while attaining the best possible statistical precision. The goal of $0.1 \%$ RSD precision for $>100 \mathrm{~g} / 2$ requires high counting rates. The pasiive rate from the samples is limited by nature, but nigher counting rates from the transmission source can allow a greater fraction of the 1000-5 count time to be devoted to the passive sample measurement.

\section{TABLE II}

AVERAGE EMPTY BOTTLE TRANSMISSION AT $186 \mathrm{keV}$

(25-Bottle Sample)

\section{High-Level Bottles}

(Sma 11)

Transmission

Std. Dev.
0.9593

0.0074
Low-Level Bottles

(Large)

0.9574

0.0019 
The measurement sequence chosen was that typically called a "two-pass assay." In this approach the shutter is opened and the transmission source is counted. Only the transmission data are analyzed from this spectrum. Then, in a second pass, the shutter is closed and the passive signal from the ${ }^{235} \mathrm{U}$ sample is counted without interference from the transmission source. The total 1000-s count time is divided between the two measurements in a fashion to be described later (see section V.0.). However, it is clear tha'c the more time devoted to the passive measurement, the better the final statistical precision will be, provided the precision of the transmission measurement is also adequate. This requires counting at high rates during the transmission measurerent to obtain the best precision in a minimum time. High rates also require a rate high enough in the $60-\mathrm{keV}{ }^{241}$ Am reference source peak to prevent its statistical precision from contributing significantly to the overall assay precision. These requirements lead to stronger ${ }^{169} \mathrm{Yb}$ transmission sources, stronger ${ }^{241}$ Am reference sources, and gross high-level system count rates in the range of $50-100 \mathrm{kHz}$.

To handle the higher counting rates, several electronic improvements were made over previous systems. A Canberra Series 90 multichannel analyzer (MCA) was used because it could handle higher counting rates ard had less dead time (a shorter cycle time) than older units. The total count rate capability of the MCA was of some concern because two detectors would be operating into a single MCA.

Most of the pileup and dead-time losses arise in the main amplifier. shorter time constants can significantly reduce these losses. We found that a 1-us amplifier time constant allowed adequate resolution and minimized pileup losses. For shorter time constants the resulution was unacceptable. We also investigated the use of a gated integrator amplifier. For our specific measurements, time constants short encugh to increase throughput (over the l-us conventional amplifier) caused the resolution to become unacceptably poor. The gated integrator amplifier is designed to make its contribution for higher-energy gamma rays, in excess of the 60-200 kev energy range used in this system.

If the statistical precision requirement $(0.1 \%)$ had been less stringent, we wuld have preferred the better resolution of a $2-\mu s$ time constant, which could potentially improve count-rate-dependent measurement biases (to be discussed later in more detail). However, the poorer resolution of the 1-us 
time constant affords two advantages that tend to reduce count-rate-dependent biases: (1) a nore uniform resolution vs energy curve, which should improve pileup corrections, and (2) a more unicorm resolution vs count-rate curve, which helps give more consistent peak area determinations using the region-ofinterest (RII) summation tecinnique.

A fast, 450-MHz clock rate, wilkinson type ADC coupled with data collection in only 1024 channels reduced dead time from the ADC digitization process. In fact, under these conditions the presence of the $A D C$ is transparent to the sysiem. Conversion and storage into ch 941 (the 198-kev $169 \mathrm{yb}$ peak) takes $2.8 \mu \mathrm{s}$, less than the $-3 \mu \mathrm{s}$ it takes the amplifier output to return from its peak to the baselirie.

The final electronic improvement was the use of new generation digital gain and zero stabilization modules, which required only 1 NIM width; older units required 4 NIM widths. With this module all the alectronics for two detectors fit into a single Nlin bin. The module also enabled us to computercontrol the stabilizer settings, a necessary feature for the sophisticated, two-pass measurement procedure.

\section{Count-Time Optimization}

Optimizing the division of the total counting time between the shutter: open and shutter-closed measurements is desirable because (1) passive: count rate varies with solution concentration, and (2) transmission count rate varies with time (32-day half-life of ${ }^{169}$ Yb transmission source) and with solution concentration.

The sample assay is proportional to the product of two independent factors: (1) the total corrected passive 186-kev count rate (corrected for pile-up and dead time), and (2) the sample attenuation correction factor. If the statistical precision of these two factors is known for a given count time, then an expression can be derived that gives the division of the total counting time that minimizes the statistical precision of the sample assay. Implementing this approach leads to a modified assay sequence.

1. Close shutter, count passive sample $100 \mathrm{~s}$, and save data in MCA.

2. Open shutter and count transmission source for $100 \mathrm{~s}$.

3. Compute precision of corrected passive rate and attenuation correction factor from the 100-s measurements. Use these in algorithm to obtain ootimum count-time division. 
4. Continue open-shutter count of transmission source (if rieeded) for remainder of optimum count time.

5. Close shutter, recall passive data, and continue passive sample count for remainder of its optimum fraction.

6. Compute final assay result.

This sequence requires no additional siutter movement nor any operator entry of count times. The total count time is obtained from a computer file. Figure 4 shows a typical optimum count-time variation for solution samples of $93 \%$ ${ }^{235} \mathrm{U}$ using a well-decayed ${ }^{169} \mathrm{Yb}$ transmission source. This curve shows that a $100-\mathrm{g} / \mathrm{\ell}$ sample is counted for $250 \mathrm{~s}$ shutter open and $750 \mathrm{~s}$ shutter closed, given a fixed 1000-s total count time. This curve will shift as the 169 Yb source decays.

This count-time optimization applies primarily to high-level solutions. For low-level solutions below about $0.1 \mathrm{~g} / \mathrm{l}$, the division is nearly always $100 \mathrm{~s}$ shutter open and $900 \mathrm{~s}$ shutter closed.

\section{E. Background Reduction}

Some special background reduction methods were necessary to improve detection sensitivity for very low concentration solutions $(<1 \mathrm{mg} / \mathrm{l})$. We found that background in the low-level head in the assay configuration was about 5-6 times that in a similar shielded detector not set up for solution assay. Background means the counts in the background continuum in the 186-keV peak region, Lypically a 3.5-kev-wide window centered at $185.7 \mathrm{keV}$. Several sources of background were identified:

1. The most obvious is natural background.

2. The americium reference source, taped to the detector end cap, contributes background from weak gamma rays with energies $>186 \mathrm{keV}$.

3. The ${ }^{169} \mathrm{Yb}$ transmission source has gammas up to $300 \mathrm{keV}$, which can penetrate the tungsten shutter.

4. Activated impurities in the $\mathrm{Yb}_{2} \mathrm{O}_{3}$ and its aluminum capsule can produce high-energy gamma rays that can easily penetrate the shutter. High-energy ganma rays from iron, nickel, zinc, manganese, cobalt, scandium, europium, and lutetium have been observed. 


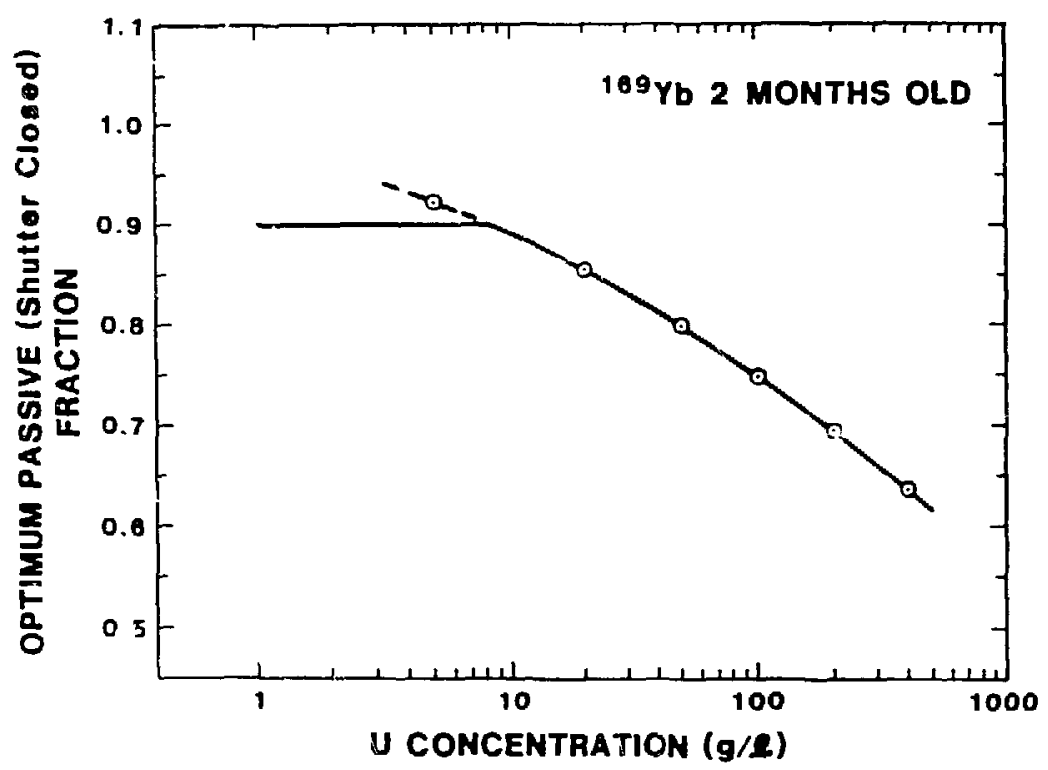

Fig. 4. Typical variation of oprimum count-time division as a function of concentration.

We took the following steps to reduce background from these sources:

1. Two-in.-thick, high-purity lead shielding reduced natural background. The same shield is also used for high-level detectors.

2. A weaker ${ }^{241}$ Am reference source is used for the low-level detectors. The waker source does not cause precisian problems because the measurement precision, in this case, is completely dominated by the $186-k e v^{235} \mathrm{u}$ count rate.

3. We increased the tungsten shutter thickness from 0.75 in. to 1.0 in. on the low-level heads.

4. We used ultra-pure $\mathrm{YD}_{2} \mathrm{O}_{3}(99.999 \%)$ in the source capsules.

5. We fabricated the source capsules from research grade, ultra-bighpurity aluminum $(99.9995+\%)$.

6. We used a larger collimator opening to allow a lower ytterbium activity and thus less impurity activation.

The ultra-high-purity aluminum in the source capsule seemed to provide the greatest background reduction when coupled with the increased shutter thickness.

Figure 5 compares pulse-height spectra from empty capsules of pure aluminum ( $99.9 \%$, which is 10 times more pure than standard 1100 aluminum) and the 


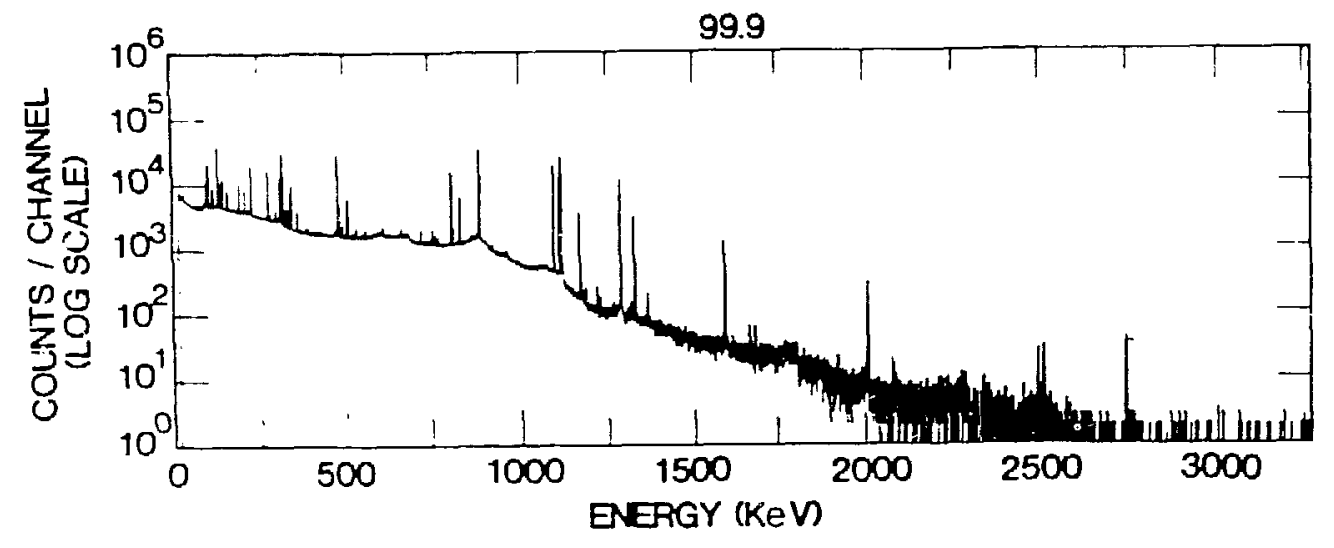

(a)

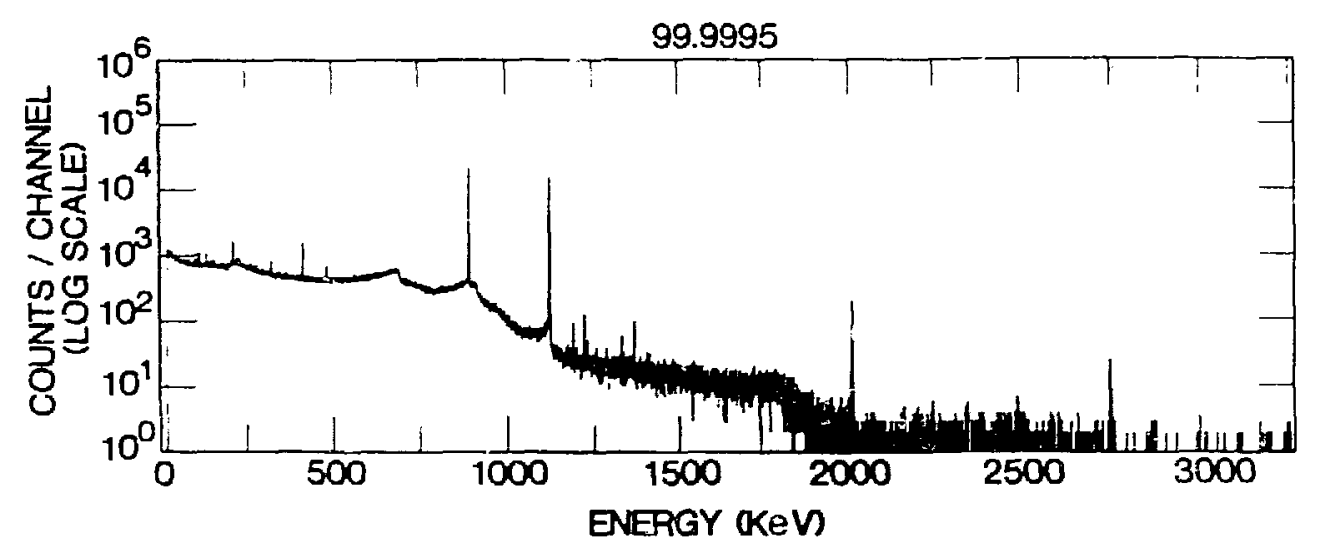

(b)

Fig. 5. Pulse-height spectra from irradiated, empty aluminum source capsules: (a) $99.9 \%$ pure aluminum and (b) $99.9995 \%$ pure a luminum.

ultra-pure aluminum (99.9995\%) that was ultimately used. Note the great reduction in gamma rays from activation products. The only major activation product left in the ultra-high-purity spectrum is 84 -day ${ }^{46} 5 \mathrm{c}$ with gamma rays at 889 and $1120 \mathrm{keV}$. Their sum peak also shows at $2009 \mathrm{kgV}$. A weak 1 ine from $15 \mathrm{~h}$ 24 iva is also seen at $2754 \mathrm{keV}$.

The steps described above reduced the background continuum from $\sim 0.25$ count/s to 0.05 count/s in $186-k e V$ peak window. This compares very favorably with the $\sim 0.04$ counts/s observed in heavily shielded detectors not set up for solution assay. The 0.05 -counts/s background gives a 3 , 1000-s detection limit of about $0.1 \mathrm{mg} 235 \mathrm{U} / \mathrm{\ell}$ in a 200-me sample. These background count rates at Los Alamos (2200 maltitude) are about twice those at sea level. 


\section{F. Transmission Correction}

Accurate transmission or attenuation corrections accounting for selfabsorption of the assay gamma ray in the sample are essential for $0.1 \%$ measurement accuracy. Sample transmissions at $186 \mathrm{keV}$ vary over a fairly wide range. Low-level solutions have a transmission of around 0.45 , independecit of uranium roncentration, which represents the attenuation in $5.7 \mathrm{~cm}$ of weak nitric acid. The transmission of high-level solutions ranges from 0.3 at $400 \mathrm{~g} / \mathrm{l}$ to about 0.8 at $1 \mathrm{~g} / \mathrm{e}$.

The transmission-correction formalism uses a numerical integration technique that accounts for the finite extent of both the sample and detector. A procedure developed hy $c$ line ${ }^{3}$ characterizes the detector efficiency as a function of energy and source position. This characterization was done once for a detectur similar to those used in this system. The same efficiency parameters are used for both high-and low-level detectors. The primary parameters needed for the correction are samp?e radius, sample depth, sample transmission, and sample-tc-detector distance. The requirement for accurate knowledge of the sclution depth arises from its use in this correction factor calculation, which takes about $12 \mathrm{~s}$ on a Micro PDP/11 computer.

The relative error in the transmission-correction factor is about 0.4 times the relative error in the measured solution transmission. This eases the accuracy requirements for transmission measurement.

\section{G. Solution Depth Measurement}

As of this writing the solution depth measuring device had not been comoleted, so its effects on the measurement accuracy have not been assessed. Refer to section IV.A. (discussion on the up-looking detector configuration) and to Ref. 2, which states that the measured sample depth must be within 0.003 in. of its trise vilue to keep assay errors below $0.1 \%$ (at a 3-cm sampleto-detector distance).

\section{PERFORMANCE TESTING}

A. High-Level Detectors

Over a period of a year, we performed tens of thousands of measurements on about 60 different sclution standards to characterize the ALSAS performance. 
In addition to characterizing the system performance, we learned another valuable and unexpected lesson. It is difficult to obtain uranium solutions with relative uranium mass values accurate to better than $0.1 \%$.

For accurate calibration, the ALSAS needs standards made in ar analytical chemistry laboratory. The system can measure relative ${ }^{235} \mathrm{U}$ mass of different solutions of similar concentration with extremely high precision ( $<0.05 \%$ RSD) under laboratory conditions. Calibration standards are necessary to place these measurements on an absolute scale.

The ALSAS calibration constant is expressed in units of total corrected count rate at $186 \mathrm{keV}$ per gram ${ }^{235} \mathrm{U}$. This constant is not expected to vary as a function of solution concentration. Any variation fron a constant value should be small (a few tenths of a percent) and monotonic with concentration. When we observe variations of $\geq 0.5 \%$, we first suspect two items: (1) bottle variations and (2) errors in the solution standards. It is difficult to unravel these two effects, but we can try by measuring the standards at large sample-to-detector distances $(10-20 \mathrm{~cm})$, "far-field" geometry measurements. Reference 2 shows that $1 / r^{2}$ effects from variations in bottle dimensioris and solution depths significantly decrease as the sample-to-detector distance increases. If the same relative pattern among standards persists in far-field measurements, the solution standards are probably in error. If the pattern changes as the samples are moved closer to the detector, a bottle variation error is probably present.

These far-field miasurements are extremely lengthy. Obtaining a statistical precision of $<0.1 \%$ RSD for a concentration of $10 \mathrm{~g} / \mathrm{l}$ may take several days of measurement time. Measurements at $20 \mathrm{~cm}$ are impractical at concentrations below $10 \mathrm{~g} / \mathrm{l}$.

Table III shows the measurement results on the first set of high-level solution standards. All data are normalized to the value at $350 \mathrm{~g} / \mathrm{l}$. The relative values of the system calibration constant are listed. We make the following observations:

1. For larger distances there does not seem to be a significant monotonic drift as a function of concentration.

2. For 10,15 , and $20 \mathrm{~cm}$, at a constant concentration, the values are consistent within estimated uncertainties. The 5-cm data may show bottle effects. These bottles were known to be more variable than desired. 
TABLE III

\section{RELATIVE VALUES OF SYSTEM CALIBRATION CONSTANT STANDARD SET 1}

\begin{tabular}{rrrrrr}
\multicolumn{9}{c}{ Sample-to-Detector Distance } \\
\cline { 2 - 2 } & $\underline{5 \mathrm{~cm}}$ & $\underline{10 \mathrm{~cm}}$ & $\underline{15 \mathrm{~cm}}$ & $\underline{20 \mathrm{~cm}}$ & \% RSD \\
350 & 1.000 & 1.000 & 1.000 & 1.000 & Norm. \\
200 & 0.998 & 0.998 & 0.996 & 0.997 & $<0.1$ \\
100 & 0.992 & 0.989 & 0.990 & 0.990 & $<0.1$ \\
40 & 1.004 & 0.999 & 1.000 & 1.000 & $<0.1$ \\
10 & 1.002 & 0.993 & 0.996 & 0.995 & 0.1 \\
1 & 1.003 & 0.995 & 0.998 & 1.004 & 0.2
\end{tabular}

3. Relative to the $350-\mathrm{g} / \mathrm{l}$ standard we observe the following biases: $200 \mathrm{~g} / \mathrm{2}: \quad 0.3 \%$

$100 \mathrm{~g} / 2: \sim 7.0 \%$

$10 \mathrm{~g} / \mathrm{\ell}: \quad \sim 0.5 \%$

We attribute these biases to errors in the solutiors' standard values. Solution standard errors of $0.5-1.0 \%$ magnitude make it nearly impossib?e to characterize instrument performance at the $0.1 \%$ level.

We obtained two additional sets of solution standards, and carefully chose "matched" bottles for the standards to minimize assay errors arising from bottle variations. We measured all high-level standards in a far-field geometry as well as on two identical ALSAS systems using the normal assay geometry.

Figure 6 shows a set of measurements on the third set of solution standards. Figure 6(a) gives the results for a far-field geometry; Figs. 6(b) and (c) give results for the actual assay geometry of two identical high-level assay systems. Each data set is normalized to the unweighted average of all data points. All data sets show the same oscillatory "structure." The maximum spread in the data is about $0.8 \%$. Clearly these samples are not suitable for careful calibration at the $0.1 \%$ level.

The second set of standards gave more consistent results. Figure 7 compares results from the second set (solid squares) with those from the third set (open circles). These are measurements in the normal assay geometry. Not 


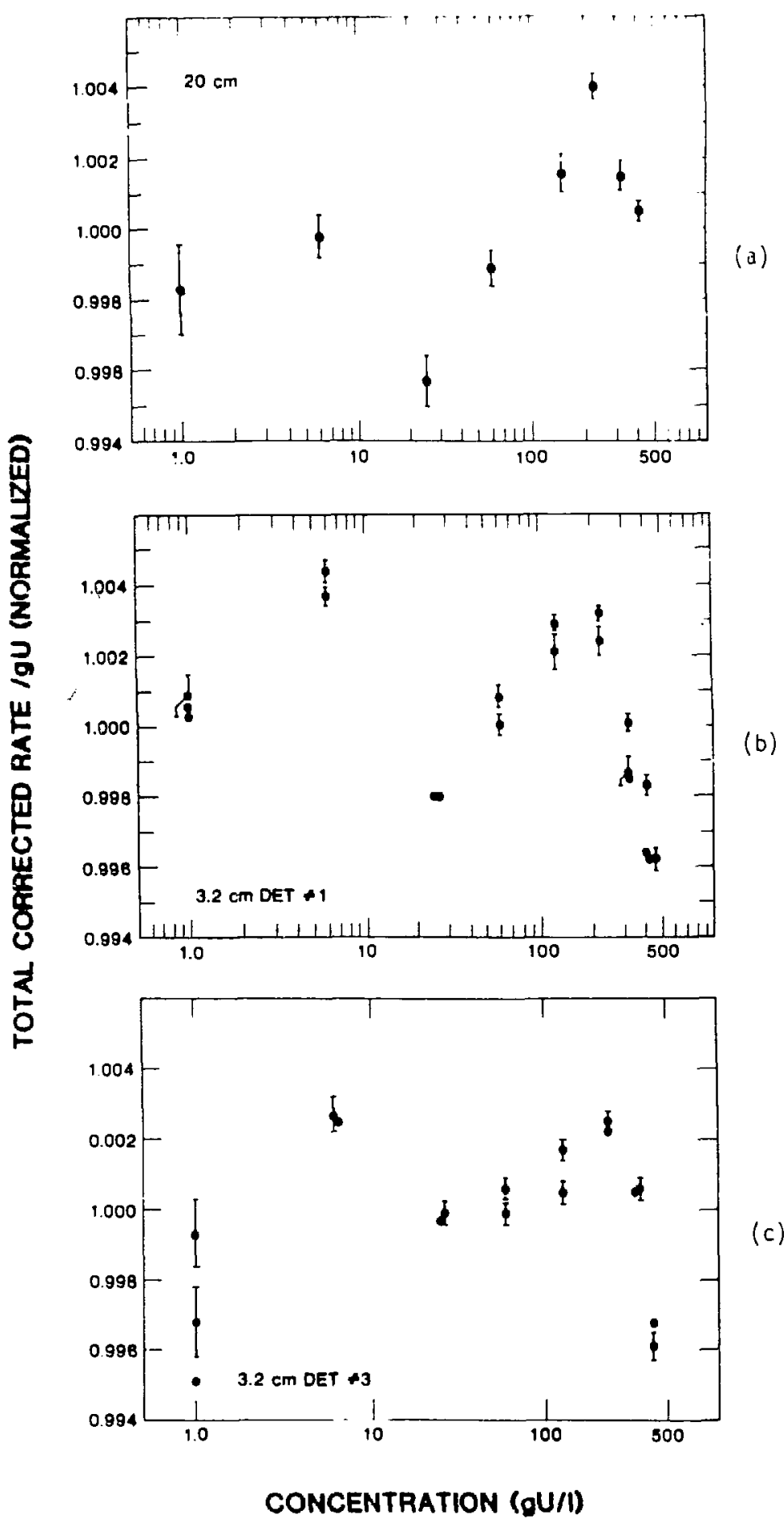

Fig. 6. Calibration constant es a function of solution concentration for the third set of calibration solutions: (a) far-field measurements at $20 \mathrm{~cm}$; (b) and (c) regular assay geometry results from two identical systems. 


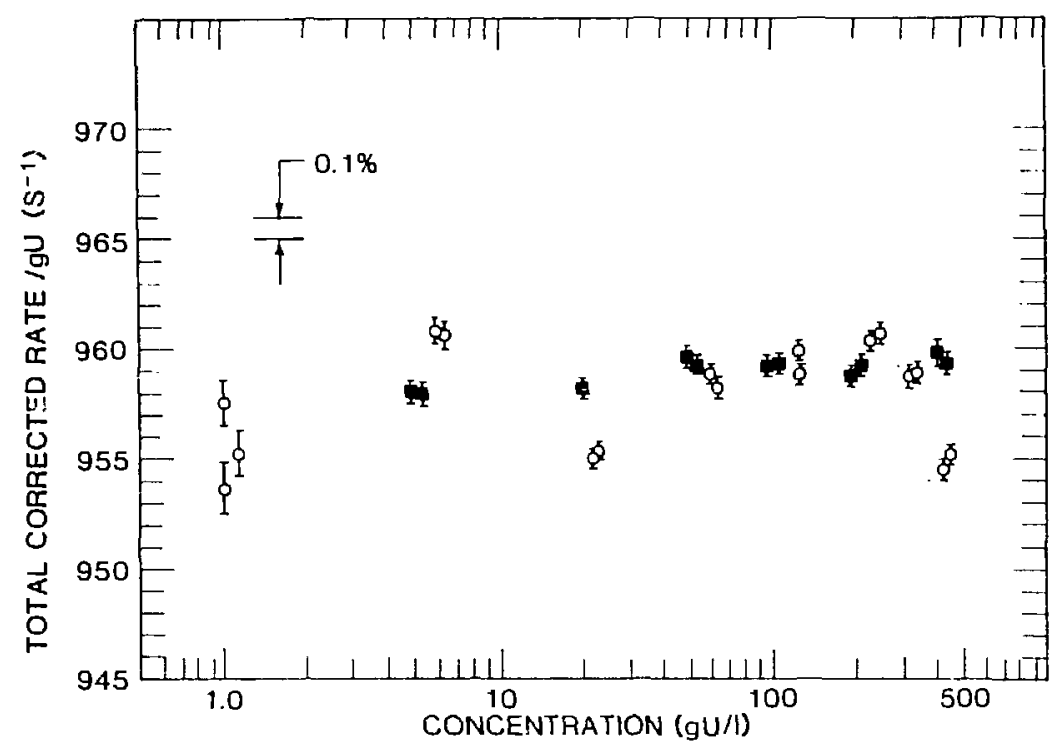

Fig. 7. Comparison of data from two calibration solution sets. Solid squares are set no. 2; open circles are set no. 3 , the same set used in fig. 6 .

only does set 2 seem to be consistent (max spread $<0.2 \%$ ), it indicates which standards in set 3 are likely to be in error, that is, $6.3 \mathrm{~g} / \ell, 20 \mathrm{~g} / \ell$, and $400 \mathrm{~g} / \ell$. These data from set 2 a 150 indicate that the goal of $0.1 \%$ accuracy is achievable under careful conditions; all bottles were hand selected and the solution depth was known and constant. These conditions are not likely to be present in routine use.

\section{B. Calibration Curve Nonlinearity}

We saw some evidence that the calibration curve may droop or "tilt." Figure 8 shows such a data set. Because this nonlinearity is monotonic, it is not obvious that the problem arises from solution standard errors. We cannot presently explain this droop, which is not always as apparent as fig. 8 would indicate. The RSD of these data points about their mean is $0.17 \%$, quite good performance by almost any standard. Nevertheless, this characteristic is bothersome because it introduces concentration-dependent biases, on the order of $0.3 \%$ at $400 \mathrm{~g} / \mathrm{e}$ in this example.

We can correct for this nonlinearity by adjusting the sample-to-detector distance parameter in the attenuation correction factor calculation (Section V.F.). Changing this distance causes the calibration curve to tilt. The data 


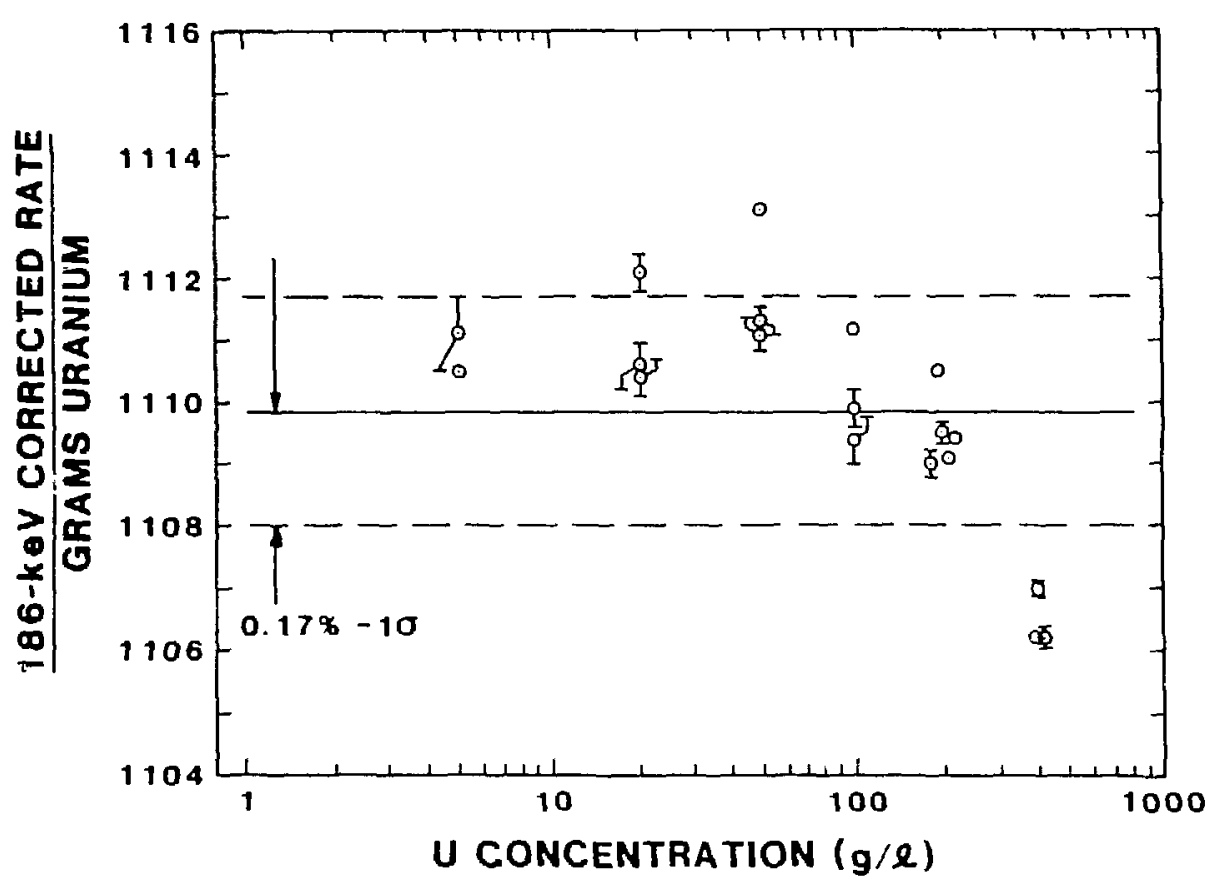

Fig. 8. High-level detector calibration data showing some nonlinearity. Sample-to-detector distance parameter $=3.2 \mathrm{~cm}$.

in Fig. 8 were computed with a value of about $3.2 \mathrm{~cm}$ for this parameter, corresponding to the physical sample-to-detector distance. If this parameter is changed to the unphysical value of $7.8 \mathrm{~cm}$, a very good correction can b: made to the Fig. 8 data. These corrected data, shown in Fig. 9, are probably the best attainable with this technique. These data show a $0.07 \%$ RSO about their mean. If we consider this a measure of system accuracy (freedom from bias), then this performance exceeds the $0.1 \%$ goal.

An additional problem surfaced, though, when we maasured high-level solutions with varying solution depths. Even though the varying sample depths were known and correctly specified in the attenuation correction factor calculation, the assay result was biased in a depth-dependent fashion. The magnilude of the bias was $\sim 7 \%$ for solution depths characteristic of 25 -me to 35 -me samples (normal solution volume is $30 \mathrm{ml}$ ). This bias appeared to arise from use of a nonphysical sample-to-detector distance parameter. When the sample-to-detector distance was returned to its physical value of $3.2 \mathrm{~cm}$, the depth-dependent bias vanished, still leaving the possibility of a nonlinear ralibration function.

We adopted the three-parameter calibration function shown in Fig. 10. These parameters ( $K, A$, and $B$ ) can easily be determined by inspection of the 20 

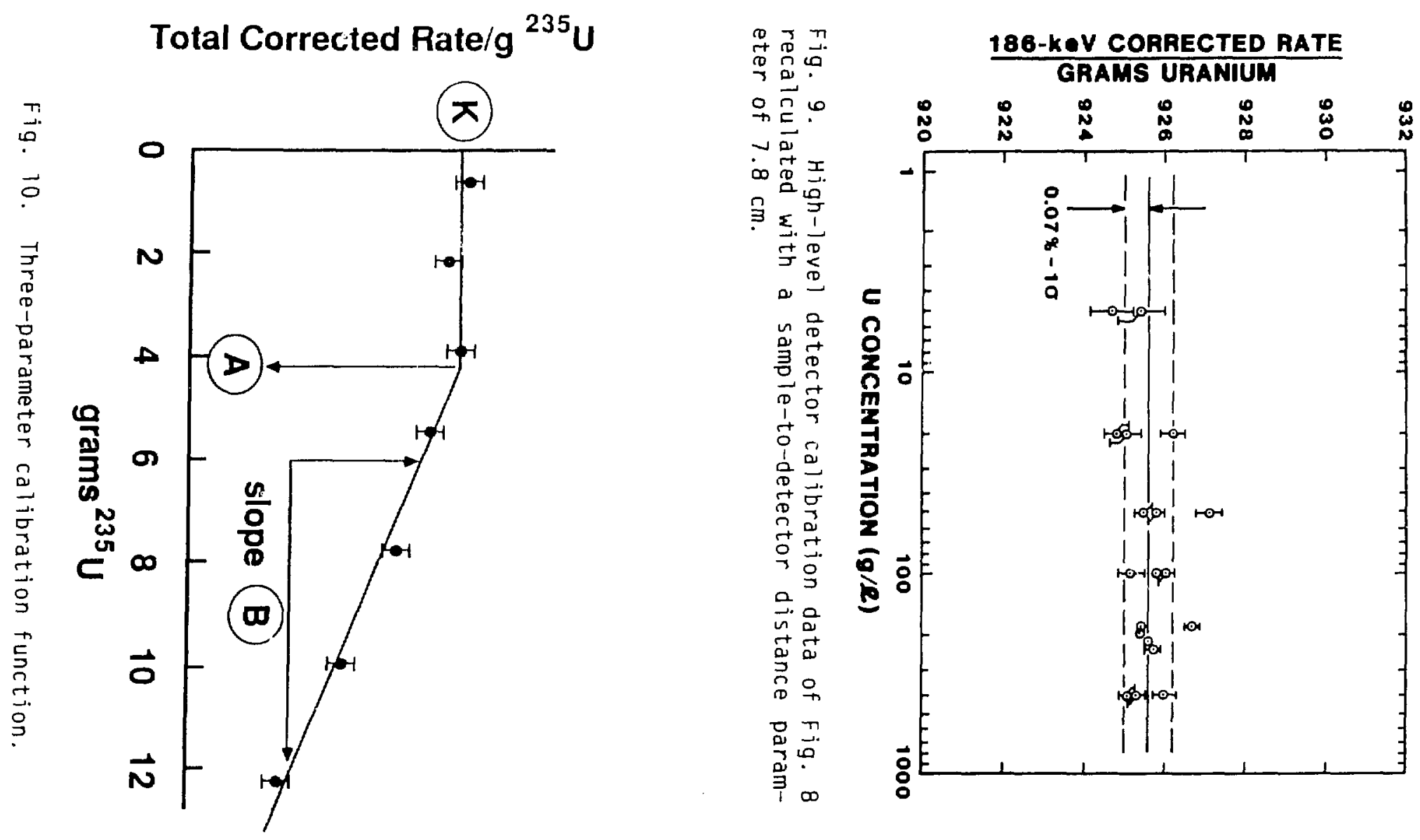
plotted data. We purposely chose this manual method over computerized fitting routines so the user would be forced to carefuily examine the data for outliers and obvious solution standard errors. This calibration function can give the same quality of $f$ it as shown in the fig. 9 tilt-corrected data, thus preserving the potential for $0.1 \%$ accuracy.

\section{Low-Level Detectors}

We expended less effort characterizing the assay accuracy of low-level detectors than the high-level detectors. For the concentration range $10^{-4}$ to $10^{-1} \mathrm{~g} \mathrm{U/ \ell}$, the 1000-s measurement precision is expected to be significantly poorer than the achievable assay accuracy. The potential assay accuracy for low-level solutions is nearly as good as that for high-level solutions; however, bottle variation effects are expected to be more severe because of the closer sample-to-detector distance $(\sim 0.5 \mathrm{~cm})$. For a constant matrix the sample transmission is independent of uranium concentration from 1 to $10^{-4} \mathrm{~g} \mathrm{U} / \mathrm{l}$, which potentially makes accurate low-level assays easier than high-level assays. Transmission variations will arise from matrix composition variations, but the effects will change the transmission only about $10 \%$.

It is also difficult to obtain accurate solution standards in the low-concentration range. Dilution techniques used to produce the samples can rapidly compound uncertainties, and analytical techniques are less accurate for low concentrations. Calibration to an accuracy of $1 \%$ RSD should be achieved. Indeed, the iow-level measuring system is probably superior to most chemical techniques in accuracy, precision, and cost for appropriate solutions.

Figure 11 shows the consistency of calibrating a low-level system over a concentration range of five orders of magnitude. Note the dual scale on the ordinate; it expands a factor of 10 between 0.99 and 1.01 . Data from two nominally identical systems are shown.

The 16 points plotted for 0.01 through $1.0 \mathrm{~g} \mathrm{U/2}$ show an RSD about their mean of less than $0.3 \%$. It is very likely that the high values for $10 \mathrm{~g} \mathrm{U} / \mathrm{e}$ result from an error in the solution standard. The measurements at $0.001 \mathrm{~g} \mathrm{U} / \mathrm{l}$ and below are fairly imprecise. The measurement accuracy at $10^{-4} \mathrm{~g} \mathrm{U} / \mathrm{e}$ is uncertain. This is at the system limit of detection and background subtraction is a large $(20 \%-50 \%)$ effect. 


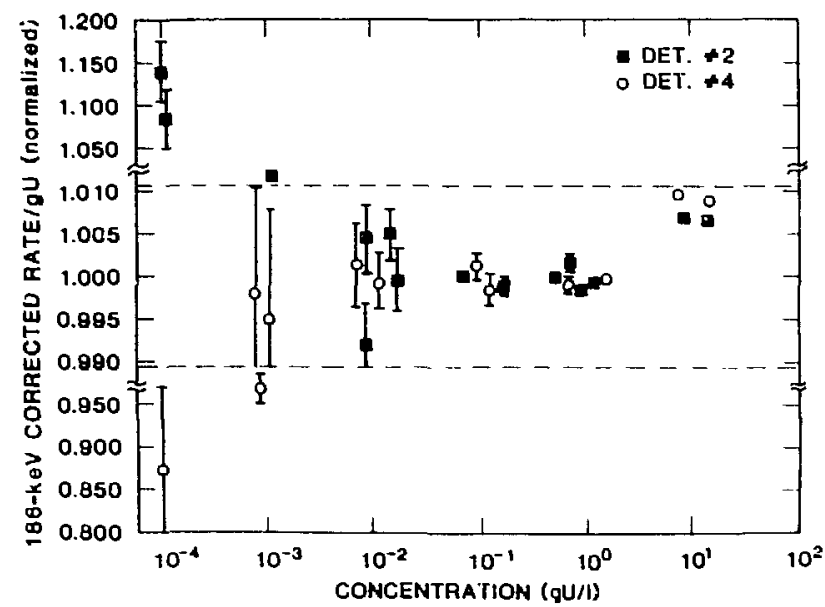

Fig. 11. Variation of calibratior constant as a function of concentration for two low-level assay systems. Dual scale on ordinate is expanded a factor of 10 between 0.99 and 1.01 .
In addition to verifying the calibration accuracy, we also verified the insensitivity of the system to fiil-height variations (as long as the variable fill height is known). Figure 12 shows the accuracy as fill height is varied over $60 \%$ for a $1 \mathrm{~g} \mathrm{U} / \mathrm{l}$ sample. Volumes over $200 \mathrm{ml}$ were not measured because at the nominal 200-me sample volume the sample bottle is nearly full.

\section{Measurement Precision}

We define assay measurement precision as pure repeatibility, where variations arise only from counting statistics. This definition is used in stating the precision of the assay result. We carefully verified tine correctness of the formulas used to propagate the counting statistics into the assay precision by comparing the observed standard deviation of repeated runs (as many as 50) with the standard deviation predicted from propagated counting statistics. We realize that this is just one component of the total measurement precision, but it is a component that can be easily verified and used as a parameter to verify the ALSAS operation in a measurement control program.

The user's goal for measurement precision was $0.1 \%$ RSD for high-concentration solutions in a 1000-s measurement time. Figure 13 displays the precision for high-level solution measurements and shows that this goal was essentially attained for concentrations $>200 \mathrm{~g} \mathrm{U} / \ell$. The data points compare the observed precision ialculated from repeated measurements with the predicted precision from propagated counting statistics. The agreement is excellent. Figure 14 shows the measurement precision as percent RSD for both high- and low-level detectors over the entire $6+$ decade measurement range.

\section{E. Count-Rate Bias Effects}

We have mentioned several effects that can bias measurements: bottle dimension changes, calibration solution errors, and incorrect sample depth 


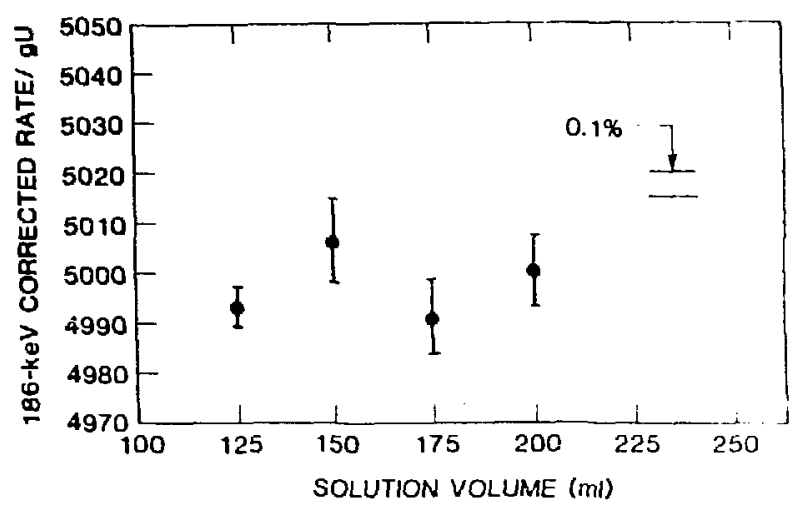

Fig. 13. Observed and predicted measurement precision for 1000-s assay cycle on high-level solutions.
Fig. 12. Immunity of low-level system to assay errors arising from known fill-height variations. Sample was $1 \mathrm{~g} \mathrm{U} / \mathrm{l}$.
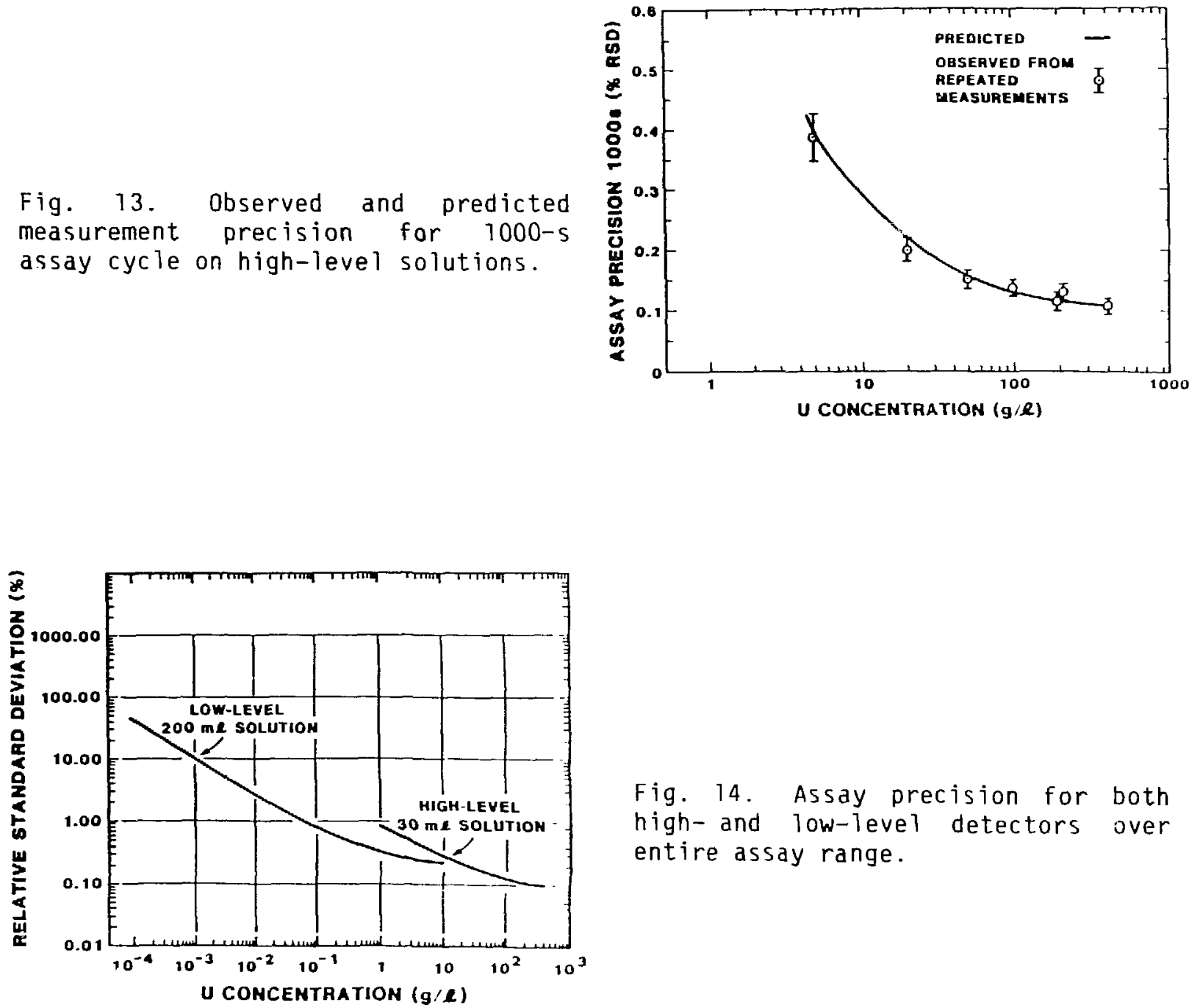

Fig. 14. Assay precision for both high-and low-level detectors over entire assay range. 
specification. Another effect may cause short-term biases. On some occasions the system calibration has shifted when the ${ }^{169} \mathrm{Yb}$ transmission source is changed. This is likely a counting-rate effect, which shows up most prominently in the measured sample transmission. With a new ytterbium source, the counting rate for the straight-through porcion of the transmission measurement may be 2-3 times its value before the source change, typically a count-rate change from $30 \mathrm{kHz}$ to $90 \mathrm{kHz}$. This causes the peak widths to broaden and may slightly bias the peak area determination. The magnitude of this effect is about $0.1-0.2 \%$.

Figures 15 and 16 explain this effect. Figure 15 shows overlaid spectra of ${ }^{169} \mathrm{Yb}$ and ${ }^{235} \mathrm{U}$ on a logarithmic scale. Figure 16 shows a combined spectrum from the shutter-open portion of a transmission run on an expanded linear scale. One can see the interferences of the 195 - and $202-k e V \quad 235 \mathrm{U}$ lines with the 198-keV ${ }^{169} \mathrm{Yb}$ line. It is difficult to avoid slight interferences nere and also difficult to find places for background RoIs for the 198-keV peak. We do not believe the direct interferfnce of the weak 198.9-keV ${ }^{235} \mathrm{U}$ peak measurably influences an assay. Relaxing the measurement precision requirements to 0.15-0.2\% would allow larger sample-to-detector distances (which would reduce bottle and sample depth effects), the better resolution of a $2-\mu \mathrm{s}$ time constant, and maybe 2048 channels of data collection. These effects would improve resolution and possibly reduce the count-rate effects.

\section{SUMMARY}

This report outlines the development of a state-of-the-art assay system for ${ }^{235} \mathrm{U}$ in solution form. The goal was to produce a system two to five times more accurate and precise than previous systems that would operate over a concentration range spanning nearly seven decades.

The sample-detector geometry greatly influences measurement precision for fixed-sample volumes. We selected an up-looking detector geometry that provided the best measurement precision for the specified sample volume. However, this selection may cause problems from sample container variability, particularly variability that affects the solution-to-detector distance.

Careful application of current generation nuclear electronics enabled us to maximize system count-rate throughput. A unique count-time optimization technique also improved the measurement statistical precision. 


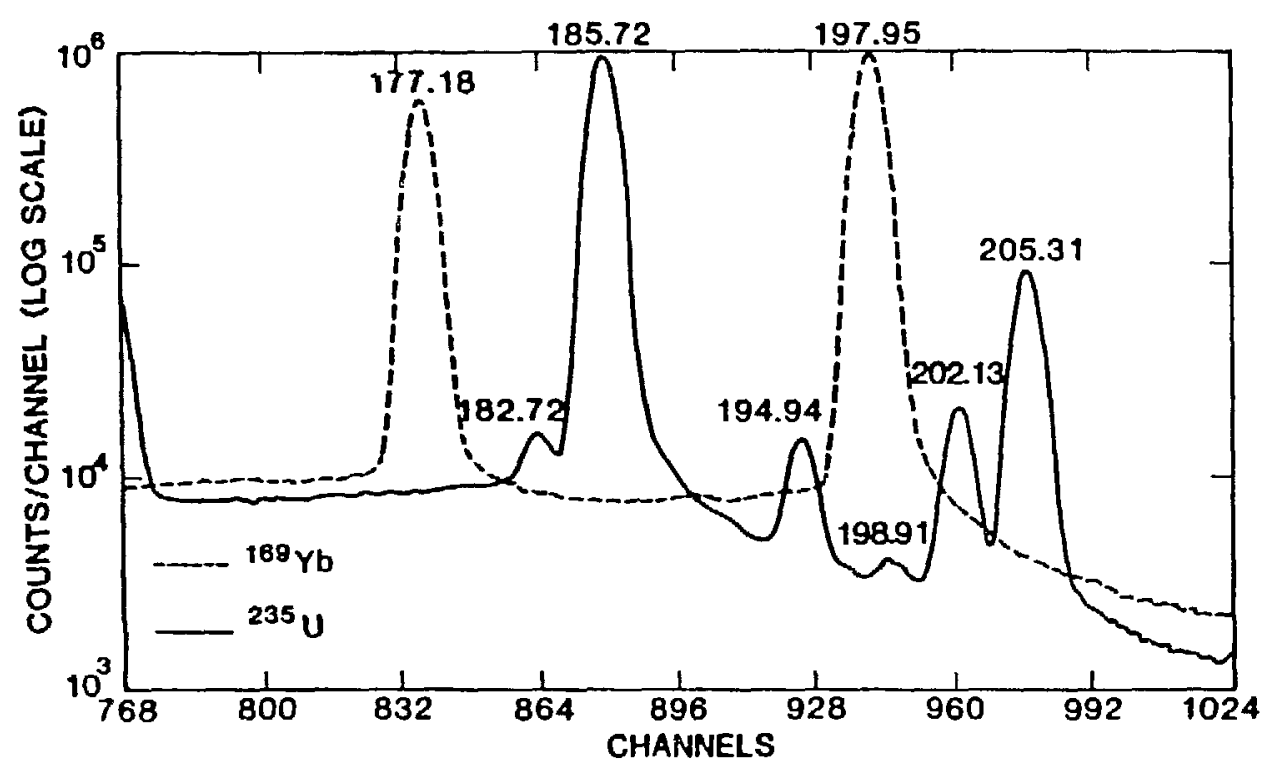

Fig. 15. Overlaid separate spectra from $235 \mathrm{U}$ and $169 \mathrm{yb}$.

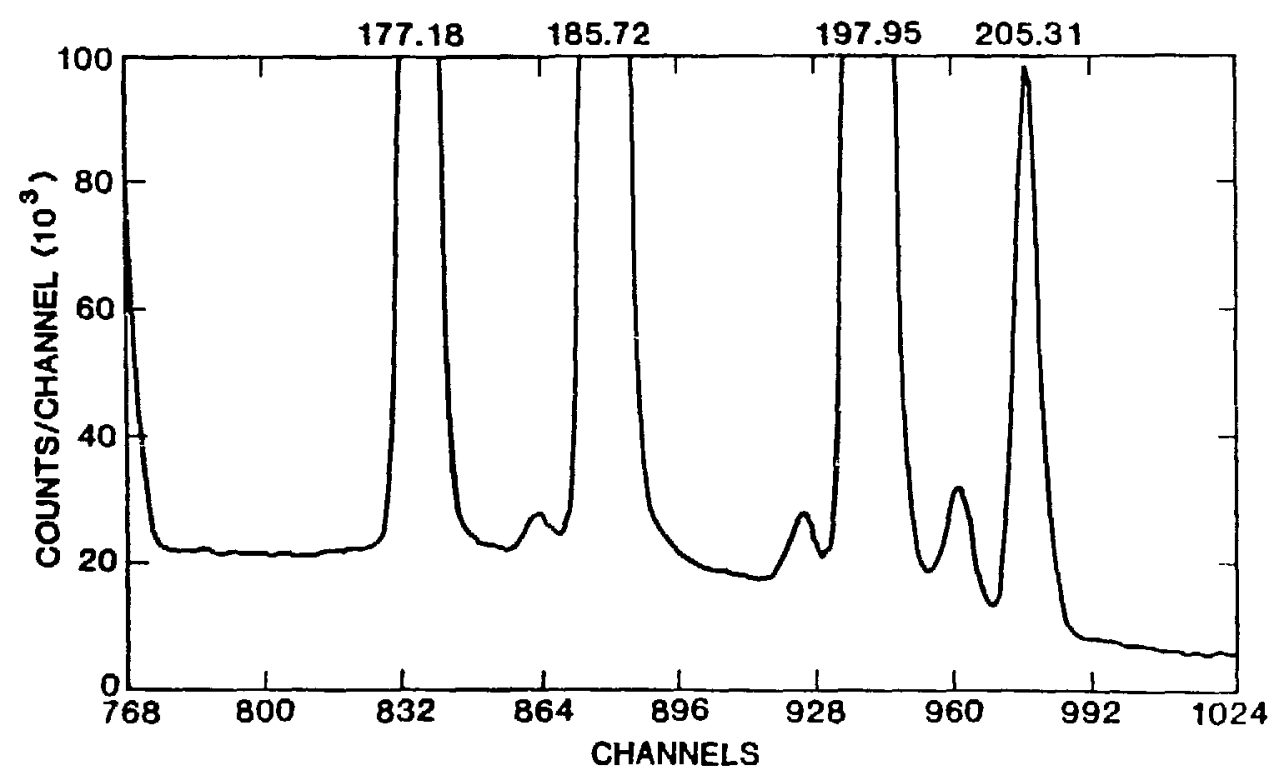

Fig. 16. Linear scale expanded portion of shutter-open part of transmission measurement showing 169 yb and $235 \mathrm{U}$ peaks. 
Several background reduction techniques redured low-level detector backgrounds to the level of natural radiation.

We applied a mure rigorous attenuation correction factor calculation than used in previous systerns.

We identified correct specification of the solution depth as necessary for accurate assays.

We spent nearly a year characterizing the ALSAS's ultimate assay accuracy. variations in chemically prepared solution standard values greatly hindered our efforts to quantify the assay accuracy. Nevertheless, under well-controlled laboratory conditions the acsay accuracy of high-level solutions $(1-500 \mathrm{~g} \mathrm{U} / \mathrm{l})$ can be $0.1 \%$ or better. In routine processing plant use, an accuracy of $\sim 0.2 \%$, similar to that expected from chemical routine analysis, should be achieved by conscientious system operators. The ALSAS is cheaper and faster than chemical analysis bui must be operated with the same care needed in chemical analysis. For low-level solutions $\left(10^{-4}-10 \mathrm{~g} \mathrm{U} / \mathrm{l}\right)$ assay accuracy can be $0.3 \%$ above $10^{-2} \mathrm{~g} \mathrm{U} / \mathrm{l}$. It should be similar for lower concentrations, but at $10^{-4} \mathrm{~g} \mathrm{U/ \ell}$ background effects may contribute to errors as large as 10\%. The minimum detectable concentration for a $200-m \ell$ sample and a $1000-\mathrm{s}$ count is $\sim 0.1 \mathrm{mg}$ $235 \mathrm{U} / \mathrm{Q}$.

Measurement precision is $\sim 0.1 \% \mathrm{RSD}$ above $200 \mathrm{~g} \mathrm{U} / \mathrm{l}$ and can be better Lhan $0.5 \%$ RSD for concentrations above $0.3 \mathrm{~g} \mathrm{~J} / \mathrm{e}$ in $1000-\mathrm{s}$ counting times.

we identified several small efferts that ran bias measurements on the order of $0.7 \%$. Slightly reduced targets for measurement precision may reduce these potential biases. This situation would probably improve overall nuclear material accountability. 


\section{APPENDIX 4}

FEATURES OF THE SYSTEM

\section{Technique}

Transmission-corrected passive gamma-ray assay is used to measure the $235 \mathrm{JJ}$ content of 200-me low-level solutions and 30-me high-level solutions. Gamma rays at $177 \mathrm{keV}$ and $198 \mathrm{keV}$ from a ${ }^{169} \mathrm{yb}$ source are used to measure the transmission through the sample. This result is used to correct a second measurement of the $185-\mathrm{keV}{ }^{235} \mathrm{u}$ gamma ray to account for self-absorption of the $185-\mathrm{keV}{ }^{235} \mathrm{U}$ gamma rays within the sample. An additional correction is made for rate-related losses caused by dead time and pulse pileup. This correction uses the known constant interaction rate of the 60-kev gamma ray from a ${ }^{241} \mathrm{Am}$ source fixed to the detector end cap. The final corrected count rate of $185-\mathrm{keV}$ gamma rays is proportional to the ${ }^{235} \mathrm{U}$ content of the sample.

\section{System Acronym}

At Line Solution Assay System (ALSAS).

\section{General}

The ALSAS consists of one rack of electronics with a single Micro PDP/ll computer controlling two detectors. Basic components for a single detector are a germanium detector, detector stand, detector shield and shutter mechanism, and the rack containing detector electronics, computer, and shutter-control electronics. The Micro PDP/l1's operating ystem is RT-11XM with separate programs for high-and low-level detectors running simultaneously. Each program has its own terminal, separate from the system console.

\section{Block Diagram}

Figure A-1 is a block diagram of the ALSAS. Only one of the two detectors is shown for clarity. The second detector would add identical comporients for the nuclear instrument module (NIM) electronics, shutter, detector, preamplifier, and liquid nitrogen ( $L N$ ) sensor. The second detector shares the shutter/ LN control, multichannel analyzer (MCA), and computer. Figure A-l shows the terminals and console for the complete two-detector system. 


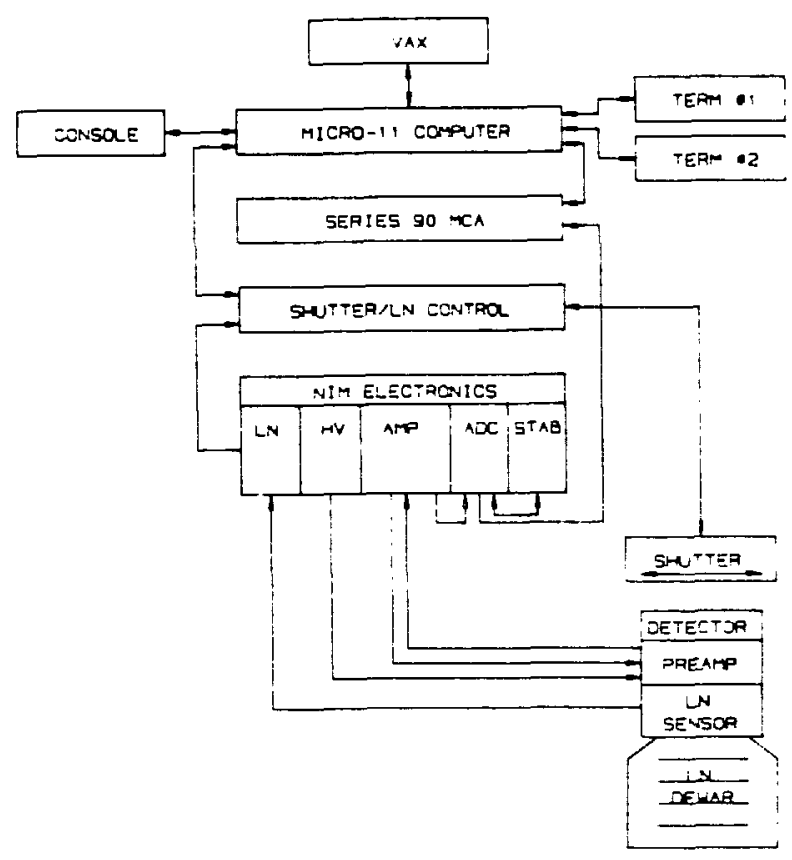

Fig. A-1. Block diagram of ALSAS showing major interconnections for one detector head.

\section{DETECTOR SPECIFICATIONS}

High Level

Model : EG\&G ORTEC Model GEM-10175-S 1

Crystal Diam: $48-50 \mathrm{~mm}$

Crystal Length : $35-45 \mathrm{~mm}$

Crystal Geometry: Closed end coaxial

Cryostat Config: Streamline vertical

End Cap Length : 7.5-8.5 in.

Dewar

: 308

Resolution

: $1332 \mathrm{keV}-<1.75 \mathrm{keV}$

$122 \mathrm{keV}-<800 \mathrm{eV}$

Peak Shape

1332 and $122 \mathrm{keV}:$ FW. $02 \mathrm{M} / \mathrm{FW} .5 \mathrm{M}<2.65^{2}$

other

\section{Low Leve 1}

EG\&G ORTEC Mode 1 GEM-25185-S

$58-62 \mathrm{~mm}$

$35-40 \mathrm{~mm}$

same

same

same

same

$<1.85 \mathrm{keV}$

$<850 \mathrm{eV}$

same

Low background internal lead shield between preamp and crysta 1

Dother detectors with equivalent specifications may be suitabie.

2 A specification of $<2.55$ would be better.

3 Internal lead shielding is present on the high-level detectors but is not required. 


\section{ELECTRONIC SPECIFICATIONS}

Liquid Nitrogen (LN) Level:

Sensor

Detector Bias Supply:

Amplifier:

Analog-to-Digital Converter:

Digital Stabilizer:

Shutter Control:

Multichannel Analyzer:

Compuier:

Operating System:

Console and Terminals:

OTHER SPECIFICATIONS

Rate-Loss Correction Source:

Transmission-Correction Source:
EG\&G ORTEC Model LNP-30-Special, dual sensors monitoring low LN level and full LN level with D729A-S dual liquid nitrogen level monitor

EG\&G ORTEC Model 459

Canberra Model 2020

Canberra Model 8077

Canberra Mode 18232

Custom module, Los Alamos National Laboratory, Group 0-1 At Line Solution Assay Power/Signal Interface

Canberra Series 90, Model 9102, two Model 9131 collect interfaces, Model 9173 high-speed intelligent $1 / 0$, Model $8673 \mathrm{~V}$ Q Bus Interface

Digital Equipment Corp. Micro/POP-11, Model 11 C23-R

$\mathrm{RT}-11 \times \mathrm{M}$

Digital Equipment Corp. Model LA120-DA

$241 \mathrm{Am}, \sim 5 \mu \mathrm{Ci}$, high level $\sim 0.5 \mu \mathrm{Ci}$, low level

Source tightly fixed to detector end cap

${ }^{169} \mathrm{Yb} 0.1 \mathrm{~g} \quad \mathrm{Yb}_{2} \mathrm{O}_{3}$ in double ultra-highpurity aluminum capsules. To be regenerated by the user. 


\section{ALGORITHMS FOR COMPUTATION OF TOTAL CORRECTED RATE}

Appendix A outlines the basic features of the data analysis. This appendix gives the details of the algorithms used to compute the total corrected rate at $186 \mathrm{keV}$, the parameter proportional to the ${ }^{235} \mathrm{U}$ mass in the sample.

The following variable arrays and definitions apply.

$\operatorname{APASIV}(E)$

ATRANS(E)

$A O(E)$

$B G(E)$
Net photopeak area of peak with energy $E$ for passive count of solution sample with shutter closed.

Net photopeak area of peak with energy $E$ for transmission measurement of solution sample with shutter open.

Net photopeak area of peak with energy $E$ for straightthrough transmission measurement, no sample, shutter open.

Net photopeak area of peak with energy $E$ for background measurement, no sample, shutter closed.

The arrays are filled up as shown below.

\begin{tabular}{|c|c|c|c|c|c|}
\hline I sotope & $\begin{array}{l}\text { Energy } \\
\text { (kev) }\end{array}$ & APASIV & ATRANS & $\underline{\mathrm{AO}}$ & $\underline{\mathrm{BG}}$ \\
\hline Am & 60 & $\operatorname{APASIV}(60)$ & ATRANS $(60)$ & $A 0(60)$ & $B G(60)$ \\
\hline Yb & 177 & & ATRANS(177) & $\mathrm{AO}(177)$ & \\
\hline$Y b$ & 198 & & ATRANS( 198$)$ & $\mathrm{A} 0(198)$ & \\
\hline U & 186 & APASIV( 186$)$ & & & $\mathrm{BG}(786)$ \\
\hline \multicolumn{2}{|c|}{ Conditions: } & $\begin{array}{l}\text { solution sample } \\
\text { shutter closed } \\
\text { passive count }\end{array}$ & $\begin{array}{l}\text { solution sample } \\
\text { shutter open } \\
\text { transmission } \\
\text { count }\end{array}$ & $\begin{array}{l}\text { no sample } \\
\text { shutter open } \\
\text { straight- } \\
\text { through count }\end{array}$ & $\begin{array}{l}\text { no sample } \\
\text { shutter closed } \\
\text { background } \\
\text { count }\end{array}$ \\
\hline
\end{tabular}


Compute sample transmission at 177 and $198 \mathrm{keV}$, TR177 and TR198.

$$
\begin{aligned}
& \operatorname{TR} 177=\operatorname{ATRANS}(177) / \operatorname{ATRANS}(60) /[\operatorname{AO}(177) / A O(60)] \\
& \operatorname{TR} 198=\operatorname{ATRANS}(198) / \operatorname{ATRANS}(60) /[\operatorname{AO}(198) / A O(60)]
\end{aligned}
$$

Both are uncorrected for ${ }^{169} \mathrm{Yb}$ source decay at this point.

Linearly interpolate measured transmissions at 177 and 198 to obtain transmission at $186 \mathrm{keV}, \mathrm{TR}$.

$$
T R=0.5891 \cdot T R 177+0.4109 \cdot T R 198
$$

Correct measured transmission for decay of ${ }^{169} \mathrm{Yb}$ source and ${ }^{241}$ Am reference source between the straight-through measurement (AO) and the solutionsample measurement (ATRANS).

$$
T R=T R \cdot E X P(-L A M \cdot T Y B D K) \cdot E X P(L Y B \cdot T Y B O K)
$$

where LAM and LYB are the decay constants of ${ }^{241}$ Am and ${ }^{169}$ Yb and TYBDK is the elapsed time between the straight-through measurement and the solution-sample transmission measurement. TR is the transmission of solution sample after an additional correction for the transmission of the empty solution container.

Correction factor for rate loss (pileup and dead-time losses) is computed for information purposes and is not used directly in this form in further calculations.

CFRL $=F E I R 60 \cdot T C L O S E / A P A S I V(60) \cdot E X P(-L A M \cdot T A M D K)$

where

CFRL = Rate-loss correction factor, a multiplicative number $\geq 1.00$.

FEIR60 = Full-energy interaction rate of $60 \mathrm{keV}{ }^{241}$ Am reference peak. This is the rate at which the 60-keV net peak area will increase in the absence of pileup and dead-time losses. Ideally, this parameter is a system constant. 
TCLOSE = shutter closed count time for passive solution-sample measurement.

TAMOK = elapsed time between last system calibration and current measurement.

Correct $186-k e v^{235} \mathrm{u}$ net peak area for background.

where

$$
\begin{aligned}
\text { A186 = } & \text { APASIV (186) }-B G(186) \cdot \operatorname{APASIV}(60) / B G(60) \cdot \operatorname{EXP}(-L A M \cdot T B G N O W) \\
\text { A186 }= & 186-k e V \text { net peak area corrected for background } \\
\text { TBGNOW = } & \text { elapsed time between background measurement (BG) and } \\
& \text { current measurement. }
\end{aligned}
$$

The total corrected rate for the $186-\mathrm{keV}{ }^{235} \mathrm{U}$ gamma ray uses the FEIR60 parameter defined previously to get familiar and meaningful units and magnitude for the result.

$$
\text { TCR186 }=\text { A186/APASIV }(60) \cdot \text { FEIR60 } \cdot \text { EXP }(-L A M \cdot T A M D K) \cdot C F A T
$$

where

$$
\begin{aligned}
& \operatorname{TCR} 186=\text { total corrected rate at } 186 \mathrm{keV} \\
& \operatorname{EXP}(\ldots)=\text { correction for decay of }{ }^{241} \text { Am reference source } \\
& \text { CFAT }=\text { correction factor for attenuation within the sample. }
\end{aligned}
$$

Finally, the sample ${ }^{235} \mathrm{U}$ mass is simply calculated from the total corrected rate by

$$
\text { GHU235 = TCR186/CALCNS }
$$


where CALCNS is the system calibration constant in units of total corrected rate per gram ${ }^{235} \mathrm{U}$.

The attenuation correction factor CFAT has been discussed in general terms in the main body of this report. It, too, is a multiplicative factor $\geq 1.00$. The sample transmission and other sample-dependent geometrical parameters are used to compute CFAT. The page of code necessary to calculate CFAT is not presented here, however, CFAT is calculated as a correction factor for an attenuating cylindrical sample with respect to a nonattenuating point source at the bottom center of the solution. 4 
APPENDIX C

SYSTEM PHOTOS 

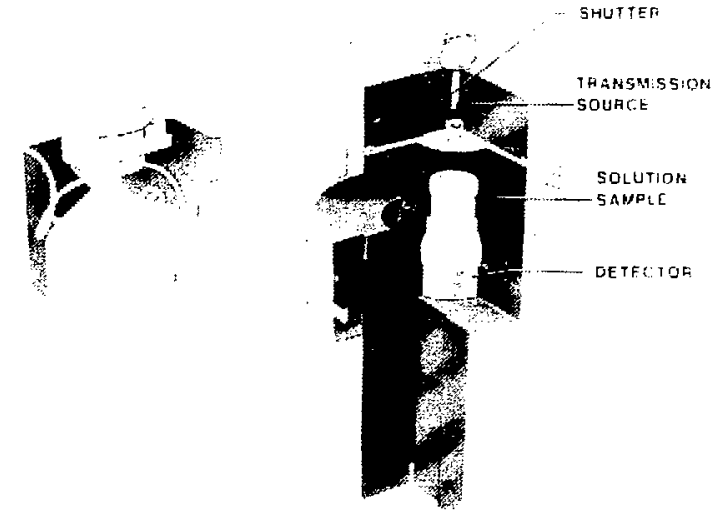

Fig. C-1. Artist conceptions of an ALSAS detector stand.

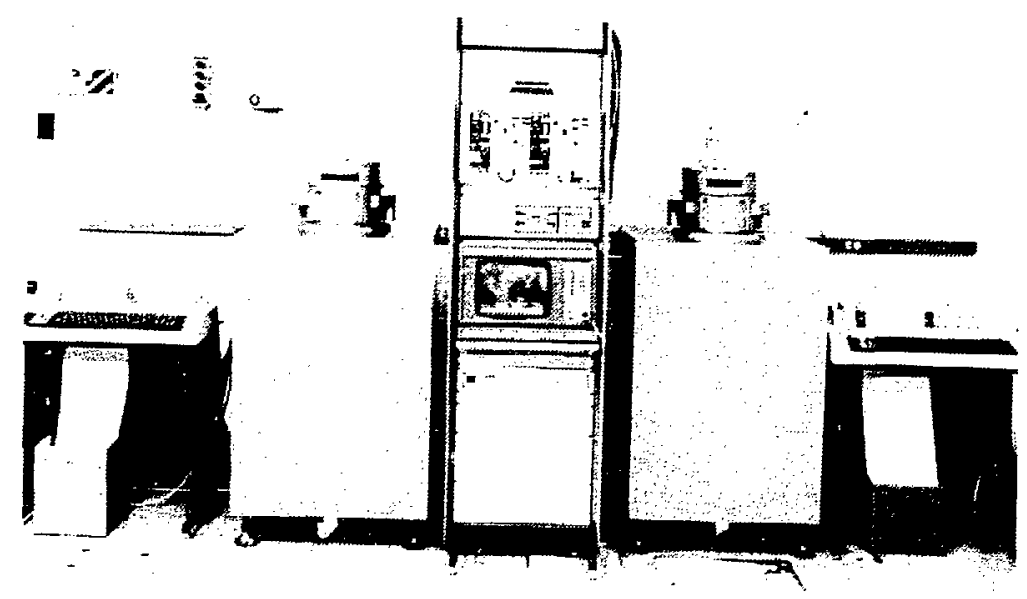

Fig. C-2. A single ALSAS system with high- and lowlevel detector heads and terminais. 


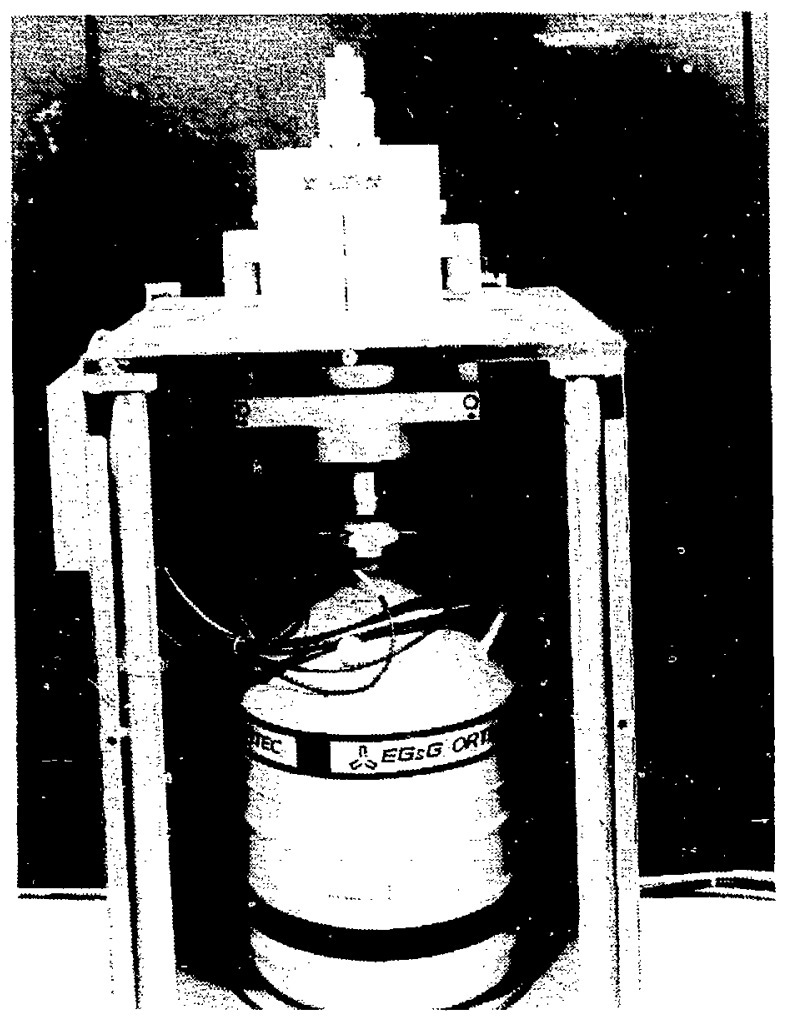

Fig. C-3. An ALSAS measurement head with front panel removed showing detector in assay position.

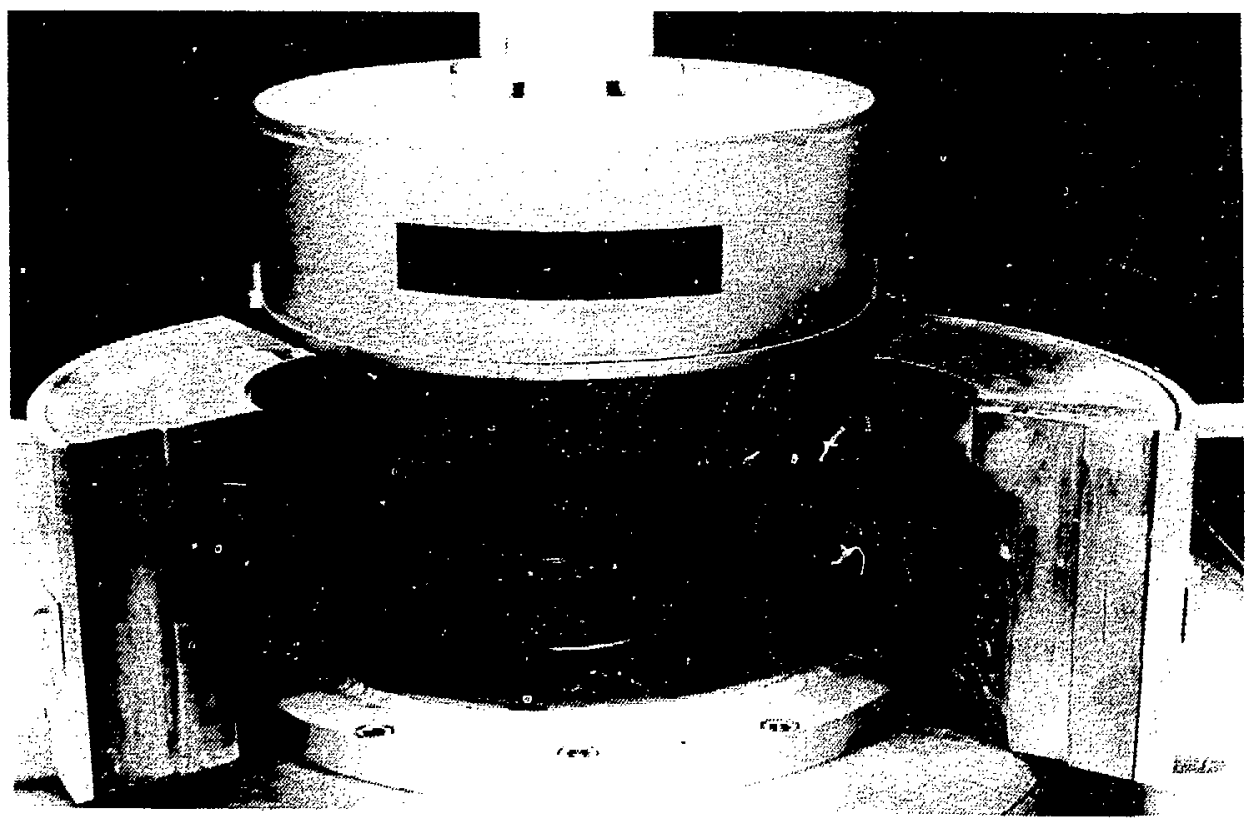

Fig. C-4. ALSAS assay chamber showing cietector end cap with 241 Am rate-loss source affixed. Solution sample holder not in place. 


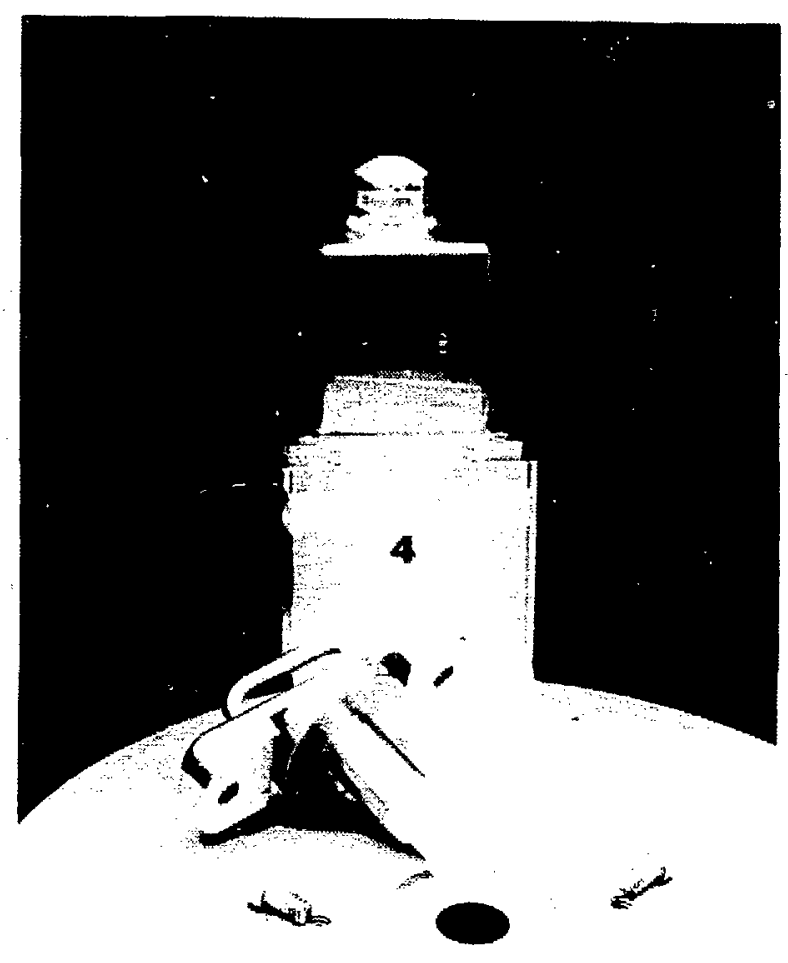

Fig. C-5. Holder for ${ }^{169} \mathrm{Yb}$ transmission source.

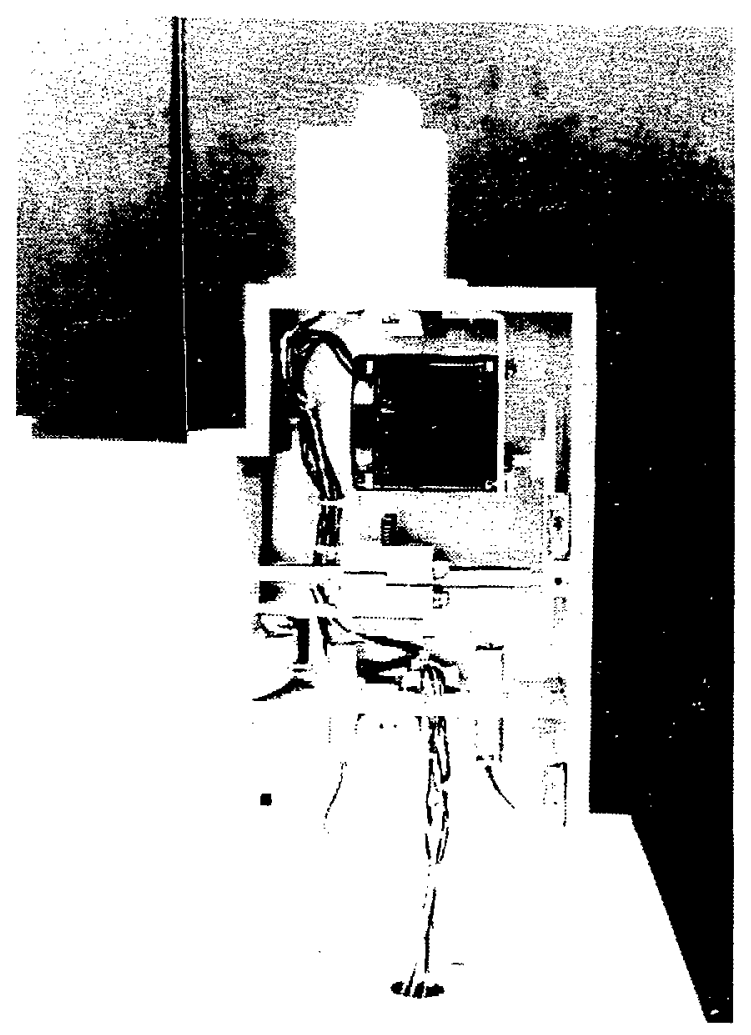

Fig. C-6. Shutter drive mechanism with side panel removed.

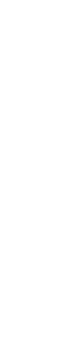




\section{REFERENCES}

1. J. K. Sprinkle, Jr., H. P. Baxman, D. G. Langner, T. R. Canada, and T. E. Sampson, "The In-Plant Evaluation of a Uranium NDA System," in Proceedings of ANS Topical Meeting (Kiawah Island, South Carolina, June 19F0), NBS Special Publication 582, pp. 324-341.

2. J. L. Parker, "Are 0.1\% - Accurate Gamma-Ray Assays Possible for 235U Solutions?" in Proc. ANS/INMM Conf. Safeguards Technol.: Process Safeguards Interfare (US DOE/New Brunswick Laboratory, August 1984), CONF-831106, pp. 142-154.

3. J. E. Cline, "A Technique of Gamma-Ray Detector Absolute Efficiency Calibration for Extended Sources," in Proceedings of the ANS Topical Conference on Computers in Activation Anzlys is and Gamma-Ray Spectroscopy (Mayaguez, Puerto Rico), CONF 780421, pp. 185-196.

4. J. L. Parker, "The Use of Calibration Standards and the Correction for Sample Self-Attenuation in Gamma-Ray Nondestructive Assay, "Los Alamos National Laboratory report LA-10045 (August 1984). 